JOURNAL OF THE

AMERICAN MATHEMATICAL SOCIETY

Volume 9, Number 3, July 1996

\title{
GEOMETRIC INVARIANT THEORY AND FLIPS
}

\author{
MICHAEL THADDEUS
}

Ever since the invention of geometric invariant theory, it has been understood that the quotient it constructs is not entirely canonical, but depends on a choice: the choice of a linearization of the group action. However, the founders of the subject never made a systematic study of this dependence. In light of its fundamental and elementary nature, this is a rather surprising gap, and this paper will attempt to fill it.

In one sense, the question can be answered almost completely. Roughly, the space of all possible linearizations is divided into finitely many polyhedral chambers within which the quotient is constant (2.3), (2.4), and when a wall between two chambers is crossed, the quotient undergoes a birational transformation which, under mild conditions, is a flip in the sense of Mori (3.3). Moreover, there are sheaves of ideals on the two quotients whose blow-ups are both isomorphic to a component of the fibred product of the two quotients over the quotient on the wall (3.5). Thus the two quotients are related by a blow-up followed by a blow-down.

The ideal sheaves cannot always be described very explicitly, but there is not much more to say in complete generality. To obtain more concrete results, we require smoothness, and certain conditions on the stabilizers which, though fairly strong, still include many interesting examples. The heart of the paper, $\S \S 4$ and 5 , is devoted to describing the birational transformations between quotients as explicitly as possible under these hypotheses. In the best case (4.8), for example, the blow-ups turn out to be just the ordinary blow-ups of certain explicit smooth subvarieties, which themselves have the structure of projective bundles.

The last three sections of the paper put this theory into practice, using it to study moduli spaces of points on the line, parabolic bundles on curves, and Bradlow pairs. An important theme is that the structure of each individual quotient is illuminated by understanding the structure of the whole family. So even if there is one especially natural linearization, the problem is still interesting. Indeed, even if the linearization is unique, useful results can be produced by enlarging the variety on which the group acts, so as to create more linearizations.

I believe that this problem is essentially elementary in nature, and I have striven to solve it using a minimum of technical machinery. For example, stability and semistability are distinguished as little as possible. Moreover, transcendental methods, choosing a maximal torus, and invoking the numerical criterion are completely avoided. The only technical tool relied on heavily is the marvelous Luna slice theorem [19]. Luckily, this is not too difficult itself, and there is a good exposition in GIT, appendix 1D. This theorem is used, for example, to give a new, easy proof of the Bialynicki-Birula decomposition theorem (1.12).

Received by the editors November 11, 1994 and, in revised form, March 23, 1995.

1991 Mathematics Subject Classification. Primary 14L30, 14D20. 
The contents of the paper are more precisely as follows. Section 1 treats the simplest case: that of an affine variety $X$ acted on by the multiplicative group $k^{\times}$, and linearized on the trivial bundle. This case has already been treated by Brion and Procesi [7], but the approach here is somewhat different, utilizing $\mathbb{Z}$-graded rings. The four main results are models for what comes later. The first result, (1.6), asserts that the two quotients $X / / \pm$ coming from nontrivial linearizations are typically related by a flip over the affine quotient $X / / 0$. The second, (1.9), describes how to blow up ideal sheaves on $X / / \pm$ to obtain varieties which are both isomorphic to the same component of the fibred product $X / /-\times_{X / / 0} X / /+$. In other words, it shows how to get from $X / /+$ to $X / /$ - by performing a blow-up followed by a blow-down. The third result, (1.13), asserts that when $X$ is smooth, the blow-up loci are supported on subvarieties isomorphic to weighted projective fibrations over the fixed-point set. Finally, the fourth, (1.15), identifies these fibrations, in what gauge theorists would call the quasi-free case, as the projectivizations of weight subbundles of the normal bundle to the fixed-point set. Moreover, the blow-ups are just the familiar blow-ups of smooth varieties along smooth subvarieties.

Sections 3, 4, and 5 are concerned with generalizing these results in three ways. First, the variety $X$ may be any quasi-projective variety, projective over an affine. Second, the group acting may be any reductive algebraic group. Third, the linearization may be arbitrary. But $\S 2$ assumes $X$ is normal and projective, and is something of a digression. It starts off by introducing a notion of $G$-algebraic equivalence, and shows, following Mumford, that linearizations equivalent in this way give the same quotients. Hence quotients are really parametrized by the space of equivalence classes, the $G$-Néron-Severi group $\mathrm{NS}^{G}$. Just as in the DuistermaatHeckman theory in symplectic geometry, it turns out (2.3) that $\mathrm{NS}^{G} \otimes \mathbb{Q}$ is divided into chambers, on which the quotient is constant. Moreover, there are only finitely many chambers (2.4).

The analogues of the four main results of $\S 1$ then apply to quotients in adjacent chambers, though they are stated in a somewhat more general setting. The first two results are readily generalized to (3.3) and (3.5). The second two, however, require the hypotheses mentioned above; indeed, there are two analogues of each. The first, (4.7) and (4.8), make fairly strong hypotheses, and show that the weighted projective fibrations are locally trivial. The second, (5.6) and (5.9), relax the hypotheses somewhat, but conclude only that the fibrations are locally trivial in the étale topology. Counterexamples (5.8) and (6.2) show that the hypotheses are necessary.

The strategy for proving all four of these results is not to imitate the proofs in the simple case, but rather to reduce to this case by means of a trick. In fact, given a variety $X$ acted on by a group $G$, and a family of linearizations parametrized by $t$, we construct in (3.1) a new variety $Z$, dubbed the "master space" by Bertram, with an action of a torus $T$, and a family of linearizations on $\mathcal{O}(1)$ parametrized by $t$, such that $X / / G(t)=Z / / T(t)$ naturally. This reduces everything to the simple case.

The final sections, $\S \S 6,7$, and 8 , are in a more discursive style; they explain how to apply the theory of the preceding sections to some examples. In all cases, the strongest hypotheses are satisfied, so the best result (4.8) holds. Perhaps the simplest interesting moduli problems are those of (ordered or unordered) sets of $n$ points in $\mathbb{P}^{1}$; these are studied in $\S 6$. The ideas here should have many applications, but only a very simple one is given: the formula of Kirwan [18] for the Betti numbers 
of the moduli spaces when $n$ is odd. In $\S 7$ the theory is applied to parabolic bundles on a curve, and the results of Boden and $\mathrm{Hu}[8]$ are recovered and extended. Finally, in $\S 8$, the theory is applied to Bradlow pairs on a curve, recovering the results of the author [27] and Bertram et al. [2].

While carrying out this research, I was aware of the parallel work of Dolgachev and $\mathrm{Hu}$, and I received their preprint [9] while this paper was being written. Their main result essentially corresponds to (5.6), except for the statement about local triviality in the étale topology. Some of the preliminary material, corresponding to my $\S 2$, also overlaps. I am indebted to them for the observation quoted just after (4.4), and for the result (2.4), though my proof of the latter is original. The results which I believe are unique to the present paper are those on flips, blow-ups, the local triviality of the exceptional loci, the identification of the projective bundles in the quasi-free case, and the examples. In any case, even where our results coincide, our methods of proof are quite different.

A few words on notation and terminology are needed. Many of the statements involve the symbol \pm . This should always be read as two distinct statements: that is, $X^{ \pm}$means $X^{+}$(resp. $X^{-}$), never $X^{+} \cup X^{-}$or $X^{+} \cap X^{-}$. Similarly, $X^{\mp}$ means $X^{-}\left(\operatorname{resp} . X^{+}\right)$. The quotient of $X$ by $G$ is denoted $X / / G$, or $X / / G(L)$ to emphasize the choice of a linearization $L$. When there is no possibility of confusion, we indulge in such abuses of notation as $X / / \pm$, which are explained in the text. The stabilizer in $G$ of a point $x \in X$ is denoted $G_{x}$. When $E$ and $F$ are varieties with morphisms to $G$, then $E \times{ }_{G} F$ denotes the fibred product; but if $G$ is a group acting on $E$ and $F$, then $E \times{ }_{G} F$ denotes the twisted quotient $(E \times F) / G$. Unfortunately, both notations are completely standard.

For stable and semistable sets, the more modern definitions of [24] are followed, not those of [23], which incidentally is often referred to as GIT. Points are assumed to be closed unless otherwise stated.

All varieties are over a fixed algebraically closed field $k$. This may have any characteristic: although we use the Luna slice theorem, which is usually said [19, 23] to apply only to characteristic zero, in fact this hypothesis is used only to show that the stabilizer must be linearly reductive. Since all the stabilizers we encounter will be reduced subgroups of the multiplicative group, this will be true in any characteristic.

By the way, most of the results in $\S \S 1$ and 3 apply not only to varieties, but to schemes of finite type over $k$. But for simplicity everything is stated for varieties.

In $\S \S 3,4$, and 5 , we assume that the variety $X$ acted on by $G$ is projective over an affine. This is the largest category in which geometric invariant theory assures us that the semistable set has a good quotient in the sense of Seshadri [24, 25]. We recall that $\pi: X \rightarrow Y$ is a good quotient if $\pi$ is affine, $G$-invariant, and surjective, satisfies $\pi_{*}\left(\mathcal{O}_{X}\right)^{G}=\mathcal{O}_{Y}$, and takes disjoint $G$-invariant closed subsets to disjoint closed subsets. Note that the last property already fails for the action of $k^{\times}$on $\mathbb{A}^{2} \backslash 0$ with weights 1 and -1 .

Finally, since the experts do not entirely agree on the definition of a flip, here is what we shall use. Let $X_{-} \rightarrow X_{0}$ be a small contraction of varieties over $k$. This means a small birational proper morphism; small means that the exceptional set has codimension greater than 1. (Warning: this appears to be the prevailing terminology in Mori theory [13, (2.1.6)], but not in intersection homology, where small is more strictly defined.) Let $D$ be a $\mathbb{Q}$-Cartier divisor class on $X_{-}$which is relatively negative over $X_{0}$; that is, $\mathcal{O}(-D)$ is relatively ample. Then the $D$-flip is 
a variety $X_{+}$, with a small contraction $X_{+} \rightarrow X_{0}$, such that, if $g: X_{-} \rightarrow X_{+}$is the induced birational map, then the divisor class $g_{*} D$ is $\mathbb{Q}$-Cartier, and $\mathcal{O}(D)$ is relatively ample over $X_{0}$. We emphasize the shift between ampleness of $\mathcal{O}(-D)$ and that of $\mathcal{O}(D)$. If a flip exists it is easily seen to be unique. Note that several authors, including Mori [22], require that each contraction reduce the Picard number by exactly 1 . We will not require this; indeed, it is not generally true of our flips [28, 4.7]. For convenience, $D$-flips will be referred to simply as flips. However, in the literature, the unmodified word flip has traditionally denoted a $K$-flip where $K$ is the canonical divisor of $X_{-}$; this is not what we will mean.

\section{The Simplest CASE}

We begin by examining the simplest case, that of an affine variety acted on by the multiplicative group of $k$. This has been studied before in several papers, that of Brion and Procesi [7] being closest to our treatment; but we will clarify, extend, and slightly correct the existing results.

Let $R$ be a finitely-generated integral algebra over the algebraically closed field $k$, so that $X=\operatorname{Spec} R$ is an affine variety over $k$. In this section only, $G$ will denote the multiplicative group of $k$. An action of $G$ on $\operatorname{Spec} R$ is equivalent to a $\mathbb{Z}$-grading of $R$ over $k$, say $R=\bigoplus_{i \in \mathbb{Z}} R_{i}$. We will study geometric invariant theory quotients $X / / G$, linearized on the trivial bundle.

So choose any $n \in \mathbb{Z}$, and define a $\mathbb{Z}$-grading on $R[z]$ by $R_{i} \subset R[z]_{i}, z \in R[z]_{-n}$. Of course $R[z]$ is also $\mathbb{N}$-graded by the degree in $z$, but this $\mathbb{Z}$-grading is different. Since $X=\operatorname{Spec} R=\operatorname{Proj} R[z]$, the $\mathbb{Z}$-grading is equivalent to a linearization on $\mathcal{O}$ of the $G$-action on $X$. The quotient is $\operatorname{Proj} R[z]^{G}=\operatorname{Proj} R[z]_{0}=\operatorname{Proj} \bigoplus_{i \in \mathbb{N}} R_{n i} z^{i}$. For $n=0$, this is just $\operatorname{Proj} R_{0}[z]=\operatorname{Spec} R_{0}$, the usual affine quotient $[24,3.5$; GIT, Thm. 1.1]. For $n>0$, Proj $\bigoplus_{i \in \mathbb{N}} R_{n i}=\operatorname{Proj} \bigoplus_{i \in \mathbb{N}} R_{i}$ by [16, II Ex. 5.13] (the hypothesis there is not needed for the first statement); similarly for $n<0$, $\operatorname{Proj} \bigoplus_{i \in \mathbb{N}} R_{n i}=\operatorname{Proj} \bigoplus_{i \in \mathbb{N}} R_{-i}$. Hence we need concern ourselves only with the quotients when $n=0,1$, and -1 ; we shall refer to them in this section as $X / / 0$, $X / /+$ and $X / /-$, respectively. Note that $X / / \pm$ are projective over $X / / 0$.

(1.1) Proposition. If $X / /+\neq \emptyset \neq X / /-$, then the natural morphisms $X / / \pm \rightarrow$ $X / / 0$ are birational.

Of course, if say $X / /-=\emptyset$, then $X / /+$ can be any $k$-variety projective over $X / / 0$.

Proof. For some $d>0 R_{-d}$ contains a nonzero element $t$. The function field of $X / / 0$ is $\left\{r / s \mid r, s \in R_{0}\right\}$, while that of $X / /+$ is $\left\{r / s \mid r, s \in R_{d i}\right.$ for some $\left.i \geq 0\right\}$. But the map $r / s \mapsto\left(r t^{i}\right) /\left(s t^{i}\right)$ from the latter to the former is an isomorphism.

Let $X^{ \pm} \subset X$ be the subvarieties corresponding to the ideals $\left\langle R_{i} \mid \mp i>0\right\rangle$ (note the change of sign), and let $X^{0}=X^{+} \cap X^{-}$; then $X^{0}$ corresponds to the ideal $\left\langle R_{i} \mid i \neq 0\right\rangle$. Also say $\lim g \cdot x=y$ if the morphism $G \rightarrow X$ given by $g \mapsto g \cdot x$ extends to a morphism $\mathbb{A}^{1} \rightarrow X$ such that $0 \mapsto y$.

(1.2) Lemma. As sets, $X^{ \pm}=\left\{x \in X \mid \exists \lim g^{ \pm 1} \cdot x\right\}$, and $X^{0}$ is the fixed-point set for the G-action.

Proof. A point $x$ is in $X^{+}$if and only if for all $n<0, R_{n}$ is killed by the homomorphism $R \rightarrow k\left[x, x^{-1}\right]$ of graded rings induced by $g \mapsto g \cdot x$. This in turn holds if the image of $R$ is contained in $k[x]$, that is, if $G \rightarrow X$ extends to $\mathbb{A}^{1} \rightarrow X$. The proof for $X^{-}$is similar. 
Hence $x \in X^{0}$ if and only if $\lim g^{ \pm 1} \cdot x$ both exist, that is, the closure of $G \cdot x$ is a projective variety in $X$. Since $X$ is affine, this means $x$ is a fixed point of $G$.

(1.3) Proposition. (a) $X^{s s}(0)=X$; (b) $X^{s}(0)=X \backslash\left(X^{+} \cup X^{-}\right)$; (c) $X^{s s}( \pm)=$ $X^{s}( \pm)=X \backslash X^{\mp}$.

Proof. Recall that $x \in X^{s s}(L)$ if for some $n>0$ there exists $s \in H^{0}\left(L^{n}\right)^{G}$ such that $s(x) \neq 0$, and $x \in X^{s}(L)$ if the morphism $G \rightarrow X^{s s}(L)$ given by $g \mapsto g \cdot x$ is proper. For $L=0, H^{0}\left(L^{n}\right)^{G}=R_{0}$ for all $n$, but this contains 1 , which is nowhere vanishing. That is all there is to (a). The valuative criterion implies that the morphism $G \rightarrow X$ is proper if and only if the limits do not exist, which together with (1.2) implies (b). For $L= \pm, H^{0}\left(L^{n}\right)^{G}=R_{ \pm n}$, so $X^{s s}( \pm)=X \backslash X^{\mp}$ follows immediately from the definition of $X^{ \pm}$. The additional condition of properness needed for $x \in X^{s}( \pm)$ is equivalent, by the valuative criterion, to $\lim g \cdot x$ and $\lim g^{-1} \cdot x \notin X^{s s}( \pm)$. But one does not exist, and the other, if it exists, is fixed by $G$, so is certainly not in $X^{s s}( \pm)$.

(1.4) Corollary. The morphisms $X / / \pm \rightarrow X / / 0$ are isomorphisms on the complements of $X^{ \pm} / / \pm \rightarrow X^{ \pm} / / 0$.

In good cases, $X^{ \pm} / / \pm$will be exactly the exceptional loci of the morphisms, but they can be smaller, even empty - for instance $X / / \rightarrow X / / 0$ in (1.10) below.

Proof. By (1.3), the sets $\left(X \backslash X^{ \pm}\right) / / \pm$and $\left(X \backslash X^{ \pm}\right) / / 0$ contain no quotients of strictly semistable points. They are therefore isomorphic.

(1.5) Proposition. There are canonical dominant morphisms $\pi_{ \pm}: X^{ \pm} \rightarrow X^{0}$ such that for all $x \in X^{ \pm}, \pi_{ \pm}(x)=\lim g^{ \pm 1} \cdot x$.

Proof. Note first that $R_{0} \cap\left\langle R_{i} \mid \pm i>0\right\rangle=R_{0} \cap\left\langle R_{i} \mid i \neq 0\right\rangle$. So $R /\left\langle R_{i} \mid i \neq 0\right\rangle=$ $R_{0} /\left\langle R_{i} \mid i \neq 0\right\rangle$ are naturally included in $R /\left\langle R_{i} \mid \pm i>0\right\rangle$ as the $G$-invariant parts. Hence there are natural dominant morphisms $\pi_{ \pm}: X^{ \pm} \rightarrow X^{0}$.

Because $\pi_{ \pm}$is induced by the inclusion of the degree 0 part in $R /\left\langle R_{i} \mid \mp i>0\right\rangle$, $\pi_{ \pm}(x)$ is the unique fixed point such that $f\left(\pi_{ \pm}(x)\right)=f(x)$ for all $f \in R_{0}$. But $f \in R_{0}$ means it is $G$-invariant, hence constant on orbits, so the same property is satisfied by $\lim g^{ \pm 1} \cdot x$, which is a fixed point in the closure of $G \cdot x$.

The next two results digress to show what the results so far have to do with flips.

(1.6) Proposition. If $X^{ \pm} \subset X$ have codimension $\geq 2$, then the birational map $f: X / /-\rightarrow X / /+$ is a flip with respect to $\mathcal{O}(1)$.

Proof. The hypothesis implies that the open sets $\left(X \backslash X^{ \pm}\right) / / \pm$in $X / / \pm$ have complements of codimension $\geq 2$. But by (1.4) these open sets are identified by $f$. Hence there is a well-defined push-forward $f_{*}$ of divisors.

For some $n>0$ the twisting sheaves $\mathcal{O}( \pm n) \rightarrow X / / \pm$ are line bundles. Indeed, they are the descents [10] from $X$ to $X / / \pm$ of the trivial bundle $\mathcal{O}$, with linearization given by $n$ as at the beginning of this section. Consequently, they agree on the open sets $\left(X \backslash X^{ \pm}\right) / / \pm$, so $f_{*} \mathcal{O}(-n)=\mathcal{O}(n)$. But $\mathcal{O}( \pm n) \rightarrow X / / \pm$ are relatively ample over $X / / 0$, so $f$ is a flip.

(1.7) Proposition. Let $Y_{0}$ be normal and affine over $k$, and let $f: Y_{-} \rightarrow Y_{+}$be a flip of normal varieties over $Y_{0}$. Then there exists $X$ affine over $k$ and a $G$-action on $X$ so that $Y_{0}=X / / 0, Y_{ \pm}=X / / \pm$. 
Proof. Let $L=\mathcal{O}(D)$, where $D$ is as in the definition of a flip. Since $Y_{ \pm}$are normal, and the exceptional loci of $f$ have codimension $\geq 2, f$ induces an isomorphism $H^{0}\left(Y_{-}, L^{n}\right) \cong H^{0}\left(Y_{+}, f_{*} L^{n}\right)$ for all $n$. The $\mathbb{N}$-graded algebras $\bigoplus_{n<0} H^{0}\left(Y_{-}, L^{n}\right)$ and $\bigoplus_{n>0} H^{0}\left(Y_{+}, f_{*} L^{n}\right)$ are the homogeneous coordinate rings of the quasi-projective varieties $Y_{-}$and $Y_{+}$, respectively, so are finitely-generated over $k$. Hence the same is true of the $\mathbb{Z}$-graded algebra $R=\bigoplus_{n \in \mathbb{Z}} H^{0}\left(Y_{-}, L^{n}\right)$. Let $X=\operatorname{Spec} R$ with the $G$-action coming from the grading. Then $X / / 0=Y_{0}$ and $X / / \pm=Y_{ \pm}$. I thank Miles Reid for pointing out this simple proof.

In order to describe the birational map $X / /-\rightarrow X / /+$ more explicitly, we will next construct a variety birational to $X / / \pm$ which dominates them both. It is admittedly true in general that any birational map can be factored into a blow-up and blow-down of some sheaves of ideals. The virtue of the present situation, however, is that these sheaves can be identified fairly explicitly, and that the common blow-up is precisely the fibred product.

Choose $d>0$ such that $\bigoplus_{i \in \mathbb{Z}} R_{d i}$ is generated by $R_{0}$ and $R_{ \pm d}$. Then let $\mathcal{I}^{ \pm}$be the sheaves of ideals on $X$ corresponding to $\left\langle R_{\mp d}\right\rangle$. Let $\mathcal{I}^{ \pm} / / \pm$on $X / / \pm$ and $\mathcal{I}^{ \pm} / / 0$ on $X / / 0$ be the ideal sheaves of invariants of $\mathcal{I}^{ \pm}$, that is, the sheaves of ideals locally generated by the invariant elements of $\mathcal{I}^{ \pm}$. Note that $\mathcal{I}^{ \pm}$are supported on $X^{ \pm}$, so that $\mathcal{I}^{ \pm} / / \pm$are supported on $X^{ \pm} / / \pm$.

For $i, j \geq 0$, let $R_{i, j}=R_{i} \cdot R_{-j} \subset R_{i-j}$.

(1.8) Lemma. The ideal sheaf $\left(\mathcal{I}^{+}+\mathcal{I}^{-}\right) / / 0$ is exactly $\left\langle R_{d, d}\right\rangle$, and its pullbacks by the morphisms $X / / \pm \rightarrow X / / 0$ are $\mathcal{I}^{ \pm} / / \pm$.

Proof. The ideals in $R$ corresponding to $\mathcal{I}^{ \pm}$are by definition $\left\langle R_{\mp d}\right\rangle$, and $\left\langle R_{\mp d}\right\rangle \cap$ $R_{0}=R_{d, d}$, so $\left(\mathcal{I}^{+}+\mathcal{I}^{-}\right) / / 0=\left\langle R_{d, d}\right\rangle$. Regard $X / / \pm$ as quotients with respect to the linearizations $\pm d$. Then $X / / \pm=\bigoplus_{i \geq 0} R_{ \pm d i}$, so for any nonzero $\sigma \in R_{ \pm d}$, $\operatorname{Spec}\left(\sigma^{-1} \bigoplus_{i \geq 0} R_{ \pm d i}\right)_{0}$ is affine in $X / / \pm$. But $\mathcal{I}^{ \pm} \cap\left(\sigma^{-1} \bigoplus_{i \geq 0} R_{ \pm d i}\right)_{0}=\sigma^{-1}\left\langle R_{d, d}\right\rangle$, so locally $\mathcal{I}^{ \pm} / \|$is the pullback of $\left\langle R_{d, d}\right\rangle$. As $\sigma$ ranges over $R_{ \pm d}$, these affines cover $X / / \pm$, so the result holds globally.

(1.9) Theorem. Suppose $X / /+\neq \emptyset \neq X / /-$. Then the blow-ups of $X / / \pm$ at $\mathcal{I}_{ \pm} / / \pm$, and the blow-up of $X / / 0$ at $\left(\mathcal{I}^{+}+\mathcal{I}^{-}\right) / / 0$, are all naturally isomorphic to the irreducible component of the fibred product $X / /-\times_{X / / 0} X / /+$ dominating $X / / 0$.

Proof. The blow-up of $X / /+$ at $\mathcal{I}_{+} / /+$is $\operatorname{Proj} \bigoplus_{n} H^{0}\left(\left(\mathcal{I}_{+} / /+\right)^{n}(d n)\right)$ for $d$ sufficiently divisible. But by $(1.8) H^{0}\left(\left(\mathcal{I}_{+} / /+\right)^{n}(d n)\right)=R_{(d+1) n, n}$, so the blow-up is $\operatorname{Proj} \bigoplus_{n} R_{(d+1) n, n}$. There is a surjection of $R_{0}$-modules $R_{(d+1) n} \otimes_{R_{0}} R_{-n} \rightarrow$ $R_{(d+1) n, n}$, so the blow-up embeds in Proj $\bigoplus_{n} R_{(d+1) n} \otimes_{R_{0}} R_{-n}$. This is precisely the fibred product $X / /-\times_{X / / 0} X / /+$, with polarization $\mathcal{O}(d+1,1)$. By (1.4) this naturally contains $\left(X \backslash X^{ \pm}\right) / / \pm$as a nonempty open set, but so does the blow-up. The blow-up is certainly irreducible, so it is the component containing $\left(X \backslash X^{ \pm}\right) / / \pm$. The proof for $X / /-$ is similar. Likewise, the blow-up of $X / / 0$ at $\left(\mathcal{I}^{+}+\mathcal{I}^{-}\right) / / 0$ is $\operatorname{Proj} \bigoplus_{n}\left(\mathcal{I}^{+}+\mathcal{I}^{-} / / 0\right)^{n}=\operatorname{Proj} \bigoplus_{n} R_{d n, d n}=\operatorname{Proj} \bigoplus_{n} R_{n, n}$. This embeds in the fibred product with polarization $\mathcal{O}(1,1)$, but by (1.4) contains $\left(X \backslash X^{ \pm}\right) / / \pm$as a nonempty open set.

The ideal sheaves $\mathcal{I}^{ \pm}$are supported on $X^{ \pm}$, so $\mathcal{I}^{ \pm} / / \pm$are supported on $X^{ \pm} / / \pm$. But they are not just $\mathcal{I}_{X^{ \pm} / / \pm}$, as the following counterexample shows. 
(1.10) Counterexample. To show that the ideal sheaves of $X^{ \pm} / / \pm$cannot generally replace $\mathcal{I}_{ \pm} / / \pm$in $(1.9)$.

In other words, the blow-up may be weighted, not just the usual blow-up of a smooth subvariety. Let $G$ act on $X=\mathbb{A}^{3}$ by $\lambda(w, x, y)=\left(\lambda^{-1} w, \lambda x, \lambda^{2} y\right)$; in other words, $w \in R_{-1}, x \in R_{1}$, and $y \in R_{2}$. Then $X / / 0=\operatorname{Spec} k\left[w x, w^{2} y\right]=\mathbb{A}^{2}$, and $X / /-=\operatorname{Proj} k\left[w x, w^{2} y, z w\right]=\mathbb{A}^{2}$, where the $\mathbb{N}$-grading of every variable is 0 except $z$, which is graded by 1 . However,

$$
\begin{aligned}
X / /+ & =\operatorname{Proj} k\left[w x, w^{2} y, z x, z^{2} y\right] \\
& =\operatorname{Proj} k\left[w x, w^{2} y, z^{2}\left(w^{2} x^{2}\right), z^{2}\left(w^{2} y\right)\right] \\
& =\operatorname{Proj} k\left[u, v, z u^{2}, z v\right],
\end{aligned}
$$

which is $\mathbb{A}^{2}$ blown up at the ideal $\left\langle u^{2}, v\right\rangle$. This has a rational double point, so is not the usual blow-up at a point.

The paper [7] of Brion and Procesi asserts (in section 2.3) a result very similar to (1.9). They state that the two quotients are related by blow-ups - "éclatements"of certain subvarieties. The counterexample above shows that the blow-ups must sometimes be weighted, that is, must have non-reduced centres. Brion and Procesi do not state this explicitly, but they are no doubt aware of it. Another minor contradiction to their result is furnished by the following counterexample.

(1.11) Counterexample. To show that the fibred product of (1.9) can be reducible, with the blow-up as one of its irreducible components.

Let $G$ act on the singular normal variety $X=\operatorname{Spec} k\left[a^{2}, a b, b^{2}, c, d\right] /\langle a d-b c\rangle$ where $a, b, c, d$ are of degree 1 , acted on with weights $1,-1,1,-1$ respectively. Then

$$
\begin{aligned}
X / / 0 & =\operatorname{Spec} k\left[a b, c d, a^{2} d^{2}, b^{2} c^{2}\right] /\langle a d-b c\rangle \\
& =\operatorname{Spec} k[a b, c d] \\
& =\mathbb{A}^{2} .
\end{aligned}
$$

But, using the same $\mathbb{N}$-grading convention as in the previous example,

$$
\begin{aligned}
X / /- & \operatorname{Proj} k\left[a b, c d, z^{2} b^{2}, z d\right] \\
& =\operatorname{Proj} k[u, v, z u, z v] \\
& =\tilde{\mathbb{A}}^{2}
\end{aligned}
$$

that is, the blow-up of $\mathbb{A}^{2}$ at the origin, and by symmetry $X / /+\cong \tilde{\mathbb{A}}^{2}$ as well. Taking $d=2$ gives ideal sheaves $\mathcal{I}_{+}=\left\langle a^{2}, c^{2}\right\rangle$ and $\mathcal{I}_{-}=\left\langle b^{2}, d^{2}\right\rangle$; the sheaf of invariants of both is $\left\langle u^{2}, v^{2}\right\rangle$, the ideal sheaf of twice the exceptional divisor. Hence blowing up $\mathcal{I}_{ \pm} / / \pm$does nothing. The fibred product, however, is $\tilde{\mathbb{A}} 2 \times_{\mathbb{A}^{2}} \tilde{\mathbb{A}}^{2}$, which is not just $\tilde{\mathbb{A}}^{2}$ : it has two components, isomorphic to $\tilde{\mathbb{A}}^{2}$ and $\mathbb{P}^{1} \times \mathbb{P}^{1}$ respectively and meeting in a $\mathbb{P}^{1}$.

Following Bialynicki-Birula [4], define the trivial $w_{i}$-fibration over a variety $Y$ to be $\mathbb{A}^{r} \times Y$, with a $G$-action induced by the action on $\mathbb{A}^{r}$ with weights $w_{i}$. A $w_{i}$-fibration over $Y$ is a variety over $Y$, with a $G$-action over the trivial action on $Y$, which is locally the trivial $w_{i}$-fibration. As Bialynicki-Birula observes, a $w_{i^{-}}$ fibration need not be a vector bundle, because the transition functions need not be linear. But if all the $w_{i}$ are equal, then it is a vector bundle.

Suppose now that $G$ acts on an affine variety $X$ which is smooth. Then it will be proved in (a) below that $X^{0}$ is also smooth. Purely for simplicity, suppose that 
it is also connected. (If not, the following theorem is still valid, but the fibrations involved need have only locally constant rank and weights.) The group $G$ acts on the normal bundle $N_{X^{0} / X}$. Let $N_{X}^{ \pm}$, or simply $N^{ \pm}$, be the subbundles of positive and negative weight spaces for this action, with weights $w_{i}^{ \pm} \in \mathbb{Z}$.

(1.12) Theorem. Suppose $X$ is smooth. Then (a) $X^{0}$ is smooth; (b) as varieties with $G$-action, $\pi_{ \pm}: X^{ \pm} \rightarrow X^{0}$ are naturally $w_{i}^{ \pm}$-fibrations; (c) $N_{X^{0} / X}$ has no zero weights, so equals $N^{+} \oplus N^{-}$; (d) the normal bundles $N_{X^{0} / X^{ \pm}}=N^{ \pm}$; (e) if all $w_{i}^{ \pm}= \pm w$ for some $w$, then $\mathcal{I}^{ \pm}=\mathcal{I}_{X^{ \pm}}^{d / w}$ and $\mathcal{I}^{+}+\mathcal{I}^{-}=\mathcal{I}_{X^{0}}^{d / w}$.

Parts (b), (c), (d) are the Bialynicki-Birula decomposition theorem [4, Thm. 4.1]. Another version of this result, possibly more familiar, gives a Morse-style decomposition of a projective variety into a disjoint union of $w_{i}$-fibrations. It follows easily from this [4].

Proof. First consider the case of a finite-dimensional vector space $V$, acted on linearly by $G$. Then $V=\operatorname{Spec} S$ for $S$ a $\mathbb{Z}$-graded polynomial algebra. This decomposes naturally into three polynomial algebras, $S=S^{-} \otimes S^{0} \otimes S^{+}$, corresponding to the subspaces of negative, zero, and positive weight. Then $\mathcal{I}^{ \pm}=\left\langle S_{\mp d}^{\mp}\right\rangle$, $V^{ \pm}=\operatorname{Spec} S^{ \pm} \otimes S^{0}$, and $V^{0}=\operatorname{Spec} S^{0}$. Parts (a)-(e) all follow easily. Indeed, the fibrations are naturally trivial.

To return to the general case, note first that if $U \subset X$ is a $G$-invariant open affine, then $U=\operatorname{Spec} F^{-1} R$ for some $F \subset R_{0}$. Hence $\left(F^{-1} R\right)_{i}=F^{-1}\left(R_{i}\right)$ for each $i$, so $\mathcal{I}_{U}^{ \pm}=\left.\mathcal{I}_{X}^{ \pm}\right|_{U}, U^{ \pm}=X^{ \pm} \cap U, U^{0}=X^{0} \cap U$, and $\pi_{ \pm}$is compatible with restriction. Consequently, the whole theorem is local in the sense that it suffices to prove it for a collection of $G$-invariant open affines in $X$ containing $X^{+} \cup X^{-}$.

Now for any closed point $x \in X^{0}$, apply the Luna slice theorem $[19,23]$ to $X$. Since $G_{x}=G$, the Luna slice is a $G$-invariant open affine $U \subset X$ containing $x$, and $G \times{ }_{G_{x}} N_{x}=T_{x} X$. Hence there is a strongly étale $G$-morphism (see $\left.[19,23]\right) \phi: U \rightarrow$ $V=T_{x} X$ such that $\phi(x)=0$. In particular, $U=U / / 0 \times_{V / / 0} V$. Any $y \in X^{+} \cup X^{-}$ is contained in some such $U$ : indeed, just take $x=\pi_{ \pm}(y)$. It therefore suffices to prove the theorem for $U$, so by abuse of notation, say $U=\operatorname{Spec} R$ from now on. The $G$-morphism $\phi$ then corresponds to a graded homomorphism $S \rightarrow R$, where $S$ is a $\mathbb{Z}$-graded polynomial ring, such that $R=R_{0} \otimes_{S_{0}} S$. In particular, $R_{i}=R_{0} \otimes_{S_{0}} S_{i}$ for each $i$. Hence $R_{ \pm d}$ and $S_{ \pm d}$ generate the same ideals in $R$, so $\mathcal{I}_{U}^{ \pm}=\phi^{-1} \mathcal{I}_{V}^{ \pm}$. Also, $U^{ \pm}=\phi^{-1} V^{ \pm}=V^{ \pm} \times_{V / / 0} U / / 0$, and $U^{0}=\phi^{-1} V^{0}=V^{0} \times_{V / / 0} U / / 0$. This immediately implies (a). Since $V^{ \pm} \rightarrow V^{0}$ are trivial fibrations, it also gives the local trivialization of $X^{ \pm} \rightarrow X^{0}$ near $x$ needed to prove (b).

Parts (c) and (d) also follow, since $\phi$, as an étale $G$-morphism, satisfies $\phi^{*} N_{V^{0} / V}=$ $N_{U^{0} / U}$ as bundles with $G$-action, so in particular $\phi^{*} N_{V}^{ \pm}=N_{U}^{ \pm}$. The hypotheses of part (e) imply that $d$ is a multiple of $w$; the conclusion holds if and only if the map $R_{\mp w}^{d / w} \rightarrow R_{\mp d}$ is surjective. This is true for $S$, and follows in general from $R_{i}=R_{0} \otimes_{S_{0}} S_{i}$.

The above methods can be used to describe the local structure of the non-reduced schemes cut out by $\mathcal{I}^{ \pm}$even when not all $w_{i}^{ \pm}= \pm w$, but we will not pursue this.

(1.13) Corollary. Suppose $X$ is smooth. Then $X^{ \pm} / / \pm$are locally trivial fibrations over $X^{0}$ with fiber the weighted projective space $\mathbb{P}\left(\left|w_{i}^{ \pm}\right|\right)$. 
If $X / /+\neq \emptyset \neq X / /-$, these are the supports of the blow-up loci of (1.9). On the other hand, if $X / /-=\emptyset$, then $X^{+}=X$ and $X^{0}=X / / 0$, so this says the natural morphism $X / /+\rightarrow X / / 0$ is a weighted projective fibration.

(1.14) Proposition. If $X$ is smooth, then $X / /-\times_{X / / 0} X / /+$ is integral.

Consequently, the blow-ups of (1.9) are precisely isomorphic to the fibred product in the smooth case.

Proof. The proof runs parallel to that of (1.12). Since the fibred product is certainly connected, and isomorphic to $X / / 0$ except over $X^{0} / / 0$, it suffices to show that it is locally integral over each point in $X^{0} / / 0$. The case of a vector space $V=\operatorname{Spec} S$ acted on linearly by $G$ is easy to check directly. In the general case, the Luna slice theorem again implies the existence of a neighborhood $U=\operatorname{Spec} R$ of each $x \in X^{0}$, and a strongly étale $G$-morphism $U \rightarrow V$ inducing $R=R_{0} \otimes S_{0} S$. The latter implies $U / / \pm=V / / \pm \times_{V / / 0} U / / 0$, so that

$$
U / /-\times_{U / / 0} U / /+=V / /-\times_{V / / 0} V / /+\times_{V / / 0} U / / 0
$$

since this is étale over $V / /-\times_{V / / 0} V / /+$, it is locally integral.

(1.15) Theorem. Suppose $X$ is smooth, and all $w_{i}^{ \pm}= \pm w$ for some $w$. Then $X^{ \pm} / / \pm$are naturally isomorphic to the projective bundles $\mathbb{P}\left(N^{ \pm}\right)$over the fixedpoint set $X^{0}$, their normal bundles are naturally isomorphic to $\pi_{ \pm}^{*} N^{\mp}(-1)$, and the blow-ups of $X / / \pm$ at $X^{ \pm} / / \pm$, and of $X / / 0$ at $X^{0} / / 0$, are all naturally isomorphic to the fibred product $X / /-\times_{X / / 0} X / /+$.

Note that if each 0-dimensional stabilizer on $X$ is trivial, then all $w_{i}^{ \pm}= \pm 1$.

Proof. All the blow-ups and the fibred product are empty if either $X / /+$ or $X / /-$ is empty, so suppose they are not. By the observation of Bialynicki-Birula quoted above, if all $w_{i}^{ \pm}= \pm w$, then the fibrations $X^{ \pm} \rightarrow X^{0}$ are actually vector bundles. But any vector bundle is naturally isomorphic to the normal bundle of its zero section, so by $(1.12)(\mathrm{d}) X^{ \pm} / / \pm=\mathbb{P}\left(X^{ \pm}\right)=\mathbb{P}\left(N^{ \pm}\right)$, and the natural $\mathcal{O}(1)$ bundles correspond. By $(1.12)(\mathrm{e}), \mathcal{I}^{ \pm}=\mathcal{I}_{X^{ \pm}}^{d / w}$. This means that $R_{\mp w}^{d / w} \rightarrow R_{\mp d}$ are surjective and hence that $\mathcal{I}^{ \pm} / / \pm=\mathcal{I}_{X^{ \pm} / / \pm}^{d / w}$ and $\left(\mathcal{I}^{+}+\mathcal{I}^{-}\right) / / 0=\mathcal{I}_{X^{0} / / 0}^{d / w}$. Since blowing up a subvariety is the same as blowing up any power of its ideal sheaf, the result follows from (1.9) and (1.14), except for the statement about normal bundles. To prove this, recall first that if $E$ is the exceptional divisor of the blow-up $\tilde{Y}$ of any affine variety $Y$ at $Z$, then $N_{E / \tilde{Y}}=\mathcal{O}_{E}(-1)$, and if $Y$ and $Z$ are smooth, then $N_{Z / Y}^{*}=\left(R^{0} \pi\right) N_{E / \tilde{Y}}^{*}$. Applying this to the case $Y=X / / 0$ shows that the normal bundle to $X^{-} / /-\times_{X^{0}} X^{+} / /+$is the restriction of $\mathcal{O}(-1,-1) \rightarrow X / /-\times_{X / / 0} X / /+$, which is exactly the obvious $\mathcal{O}(-1,-1) \rightarrow \mathbb{P} N^{+} \times_{X^{0}} \mathbb{P} N^{-}$. The normal bundle to $\mathbb{P} N^{ \pm}$is then just $\left(R^{0} \pi_{\mp} \mathcal{O}(1,1)\right)^{*}=\pi_{ \pm}^{*} N^{\mp}(-1)$.

(1.16) Example. The simplest non-trivial example of these phenomena is also the best-known; indeed it goes back to an early paper of Atiyah [1]. Let $X=\mathbb{A}^{4}$, and let $G$ act by $\lambda \cdot(v, w, x, y)=\left(\lambda v, \lambda w, \lambda^{-1} x, \lambda^{-1} y\right)$. Then $X / / 0=\operatorname{Spec} k[v x, v y, w x, w y]$ $=\operatorname{Spec}[a, b, c, d] /\langle a d-b c\rangle$, the affine cone on a smooth quadric surface in $\mathbb{P}^{3}$. But, using the $\mathbb{N}$-grading conventions of the previous examples,

$$
\begin{aligned}
X / / & =\operatorname{Proj} k[v x, v y, w x, w y, z v, z w] \\
& =\operatorname{Proj} k[a, b, c, d, z a, z c] /\langle a d-b c\rangle .
\end{aligned}
$$


This is the blow-up of $X / / 0$ at the Weil divisor cut out by $a$ and $c$. But this Weil divisor is generically Cartier, so the blow-down $X / /+\rightarrow X / / 0$ is generically an isomorphism even over the divisor. The exceptional set of the morphism therefore has codimension 2; indeed, it is the $\mathbb{P}^{1}$ lying over the cone point. Likewise, $X / /-=$ $\operatorname{Proj} k[a, b, c, d, z b, z d] /\langle a d-b c\rangle$, and similar remarks apply by symmetry. By (1.15) the fibred product $X / /-\times_{X / / 0} X / /+$ is the common blow-up of $X / / \pm$ at these $\mathbb{P}^{1}$, and also the blow-up of $X / / 0$ at the cone point. This is exactly the proper transform of the quadric cone in $\mathbb{A}^{4}$ blown up at the origin, so it has fiber $\mathbb{P}^{1} \times \mathbb{P}^{1}$ over the cone point, as expected.

\section{ThE SPACE OF LINEARIZATIONS}

In $\S \S 3,4$ and 5 we will generalize the results of $\S 1$ in three directions. First, the group $G$ may now be any reductive group over $k$. Second, the variety $X$ may now be any quasi-projective variety over $k$, projective over an affine variety. Finally, the linearization may be arbitrary. Before doing this, though, we will prove some general facts, in the case where $X$ is normal and projective, about the structure of the group of all linearizations. This will show how to apply our general results in this case.

So in this section, suppose $X$ is normal and projective over $k$, and that $G$ is a reductive group over $k$ acting on $X$. We first recall a few familiar facts about the Picard group.

In the Picard group Pic of isomorphism classes of line bundles, the property of ampleness depends only on the algebraic equivalence class of the bundle. Hence there is a well-defined ample subset $A$ of the Néron-Severi group NS of algebraic equivalence classes of line bundles. This determines the ample cone $A_{\mathbb{Q}}=A \otimes_{\mathbb{N}}$ $\mathbb{Q}_{\geq 0} \subset \mathrm{NS}_{\mathbb{Q}}=\mathrm{NS} \otimes \mathbb{Q}$. The Néron-Severi group is finitely-generated, so $\mathrm{NS}_{\mathbb{Q}}$ is a finite-dimensional rational vector space. We will refer to an element of $A$ as a polarization, and an element of $A_{\mathbb{Q}}$ as a fractional polarization.

Now let $\mathrm{Pic}^{G}$ be the group of isomorphism classes of linearizations of the $G$ action (cf. $1, \S 3$ of GIT). There is a forgetful homomorphism $f: \mathrm{Pic}^{G} \rightarrow$ Pic, whose kernel is the group of linearizations on $\mathcal{O}$, which is exactly the group $\chi(G)$ of characters of $G$. There is an equivalence relation on $\mathrm{Pic}^{G}$ analogous to algebraic equivalence on Pic; it is defined as follows. Two linearizations $L_{1}$ and $L_{2}$ are said to be $G$-algebraically equivalent if there is a connected variety $T$ containing points $t_{1}, t_{2}$, and a linearization $L$ of the $G$-action on $T \times X$ induced from the second factor, such that $\left.L\right|_{t_{1} \times X} \cong L_{1}$ and $\left.L\right|_{t_{2} \times X} \cong L_{2}$.

(2.1) Proposition. If $L$ is an ample linearization, then $X^{s s}(L)$, and the quotient $X / / G(L)$ regarded as a polarized variety, depend only on the $G$-algebraic equivalence class of $L$.

Proof. The statement about $X^{s s}(L)$ is proved exactly like Cor. 1.20 of GIT, except that the Picard group $P$ is replaced by $T$. The statement about $X / / G(L)$ as a variety then follows from this, since $X / / G(L)$ is a good quotient of $X^{s s}(L)$, hence $[24,25]$ a categorical quotient of $X^{s s}(L)$, so is uniquely determined by $X^{s s}(L)$. As for the polarization, note that, if $L_{1}$ and $L_{2}$ are $G$-linearly equivalent ample linearizations, then the linearization $L$ on $T \times X$ inducing the equivalence can be assumed ample: just tensor $L$ by the pullback of a sufficiently ample bundle on $T$. Then $\mathcal{O}(1) \rightarrow(T \times X) / / G(L)$ is a family of line bundles on $X / / G$ including $\mathcal{O}(1) \rightarrow X / / G\left(L_{1}\right)$ and $\mathcal{O}(1) \rightarrow X / / G\left(L_{2}\right)$, so these are algebraically equivalent. 
So define $\mathrm{NS}^{G}$ to be the group of $G$-algebraic equivalence classes of linearizations. In light of (2.1), by abuse of terminology an element of $\mathrm{NS}^{G}$ will frequently be called just a linearization. The forgetful map $f$ descends to $f: \mathrm{NS}^{G} \rightarrow \mathrm{NS}$.

(2.2) Proposition. This new $f$ has kernel $\chi(G)$ modulo a torsion subgroup.

Proof. Let $M \rightarrow \operatorname{Pic}_{0} X \times X$ be the Poincaré line bundle, and let $G$ act on $\operatorname{Pic}_{0} X \times$ $X$, trivially on the first factor. By Cor. 1.6 of GIT, some power $M^{n}$ of $M$ admits a linearization. Since the $n$th power morphism $\mathrm{Pic}_{0} X \rightarrow \mathrm{Pic}_{0} X$ is surjective, this shows that any element of $\operatorname{Pic}_{0} X$ has a linearization $G$-algebraically equivalent to a linearization on $\mathcal{O}$. Hence $\operatorname{ker} f$ is $\chi(G)$ modulo the subgroup of linearizations on $\mathcal{O}$ which are $G$-algebraically equivalent to the trivial linearization. We will show that this subgroup is torsion.

Suppose there is a linearization $L_{1}$ on $\mathcal{O}$ which is $G$-algebraically equivalent to the trivial linearization. Then there exist $T$ containing $t_{1}, t_{2}$ and $L$ as in the definition of $G$-algebraic equivalence. There is an induced morphism $g: T \rightarrow \operatorname{Pic}_{0} X$ such that $t_{1}, t_{2} \mapsto \mathcal{O}$. As before, let $M^{n}$ be the power of the Poincaré bundle admitting a linearization. Then $N=(1 \times g)^{*} M^{n} \otimes L^{-n}$ is a family of linearizations on $\mathcal{O} \rightarrow X$. Since the isomorphism classes of such linearizations form the discrete group $\chi(G)$, $L_{1}^{n}=N_{t_{1}}^{-1} \otimes N_{t_{2}}$ is trivial as a linearization.

Hence $\mathrm{NS}^{G}$ is finitely-generated and $\mathrm{NS}_{\mathbb{Q}}^{G}=\mathrm{NS}^{G} \otimes \mathbb{Q}$ is again a finite-dimensional rational vector space. We refer to an element of $\mathrm{NS}_{\mathbb{Q}}^{G}$ as a fractional linearization.

The map $f: \mathrm{NS}^{G} \rightarrow \mathrm{NS}$ is not necessarily surjective (see $1, \S 3$ of GIT). But by Cor. 1.6 of GIT, $f_{\mathbb{Q}}: \mathrm{NS}_{\mathbb{Q}}^{G} \rightarrow \mathrm{NS}_{\mathbb{Q}}$ is surjective. By $(2.2)$, the kernel is $\chi(G) \otimes \mathbb{Q}$, the group of fractional characters.

An ample linearization $L$ is said to be $G$-effective if $L^{n}$ has a $G$-invariant section for some $n>0$. This is equivalent to having a semistable point, so (2.1) shows that $G$-effectiveness depends only on the $G$-algebraic equivalence class of the linearization. Hence there is a well-defined $G$-effective subset $E^{G} \subset f^{-1} A \subset \mathrm{NS}^{G}$, and a $G$-effective cone $E_{\mathbb{Q}}^{G}=E^{G} \otimes_{\mathbb{N}} \mathbb{Q}_{\geq 0} \subset \mathrm{NS}_{\mathbb{Q}}^{G}$.

Now a linearization $L$ determines a quotient $X / / G$ if $L$ is ample; then $X / / G \neq \emptyset$ if and only if $L$ is also $G$-effective. Of course, we can also tensor by $\mathbb{Q}$, allowing fractional linearizations; the quotient $X / / G$ will then be fractionally polarized. Hence any fractional linearization $L \in f_{\mathbb{Q}}^{-1}\left(A_{\mathbb{Q}}\right) \subset \mathrm{NS}_{\mathbb{Q}}^{G}$ defines a fractionally polarized quotient, which will be nonempty if and only if $L \in E_{\mathbb{Q}}^{G}$. Passing to the quotient is a homogeneous process in the sense that replacing a fractional linearization $L$ by $L^{n}$ for some positive $n \in \mathbb{Q}$ has no effect on the quotient, except to replace the fractional polarization $\mathcal{O}(1)$ by $\mathcal{O}(n)$.

The first result describing the dependence of the quotient $X / / G(L)$ on the choice of $L \in \mathrm{NS}_{\mathbb{Q}}^{G}$ is the following, which is analogous to the Duistermaat-Heckman theory in symplectic geometry. A subset of $f_{\mathbb{Q}}^{-1}\left(A_{\mathbb{Q}}\right)$ will be described as polyhedral in $f_{\mathbb{Q}}^{-1}\left(A_{\mathbb{Q}}\right)$ if it is the intersection with $f_{\mathbb{Q}}^{-1}\left(A_{\mathbb{Q}}\right)$ of a polyhedron in $\mathrm{NS}_{\mathbb{Q}}^{G}$. The walls of such a subset are the intersections with $f_{\mathbb{Q}}^{-1}\left(A_{\mathbb{Q}}\right)$ of the codimension 1 faces of the polyhedron.

(2.3) Theorem. The G-effective cone $E_{\mathbb{Q}}^{G}$ is polyhedral in $f_{\mathbb{Q}}^{-1}\left(A_{\mathbb{Q}}\right)$. It is the union of a locally finite collection of cones $\bar{C}_{i}$, polyhedral in $f_{\mathbb{Q}}^{-1}\left(A_{\mathbb{Q}}\right)$ and with disjoint interiors $C_{i}$ called chambers, such that, as $t$ varies in a fixed $C_{i}$, the semistable set 
$X^{s s}(t)$ and the quotient $X / / G(t)$ remain fixed, but the fractional polarization $\mathcal{O}(1)$ on the quotient depends linearly on $t$. If $t_{0} \in \bar{C}_{i}$ and $t_{+} \in C_{i}$, then there is an inclusion $X^{s s}(+) \subset X^{s s}(0)$ inducing a canonical projective morphism $X / / G(+) \rightarrow$ $X / / G(0)$.

The proposition above could be proved directly, using the Hilbert-Mumford numerical criterion [24, 4.9; GIT, Thm. 2.1]. But it will also follow from the construction (3.1) to be introduced in the next section, so we put off the proof until then.

Theorem (2.3) asserts that the collection of chambers is locally finite; but with a little more effort we can prove a global result.

(2.4) Theorem. There are only finitely many chambers.

Proof. Suppose not. Then there exists an infinite sequence $\left\{C_{i}\right\}$ of chambers such that, for any $m, n \geq 0$, the convex hull of $C_{n} \cup C_{n+m}$ intersects the interior of $C_{n+1}$ nontrivially. Indeed, let $C_{0}$ be any chamber; then it has a wall $W_{0}$ such that there are infinitely many chambers on the other side of $W_{0}$ (or more properly, the affine hyperplane containing $\left.W_{0}\right)$. Let $C_{1}$ be the other chamber having $W_{0}$ as a wall. Inductively, given $C_{0}, \ldots, C_{n}$ such that $C_{n}$ is on the other side of $W_{i}$ from $C_{i}$ for all $i<n$, there is a wall $W_{n}$ bounding $C_{n}$ such that there are infinitely many chambers which for all $i \leq n$ are on the other side of $W_{i}$ from $C_{i}$. Let $C_{n+1}$ be the other chamber bounded by $W_{n}$. For a sequence chosen in this manner, $C_{n+m}$ is on the other side of $W_{n}$ from $C_{n}$, so the convex hull of $C_{n} \cup C_{n+m}$ meets the interior of $C_{n+1}$.

Choose an $L_{i}$ in the interior of each $C_{i}$. For any fixed $x \in X$, the set $\{L \in$ $\left.\mathrm{NS}_{\mathbb{Q}} \mid x \in X^{s s}(L)\right\}$ is convex, since $s_{ \pm} \in H^{0}\left(L_{ \pm}\right)^{G}, s_{ \pm}(x) \neq 0$ imply $s_{+} \cdot s_{-} \in$ $H^{0}\left(L_{+} \otimes L_{-}\right)^{G},\left(s_{+} \cdot s_{-}\right)(x) \neq 0$. But by $(2.3)$ it is also a union of chambers, so by induction it includes $C_{n} \cup C_{n+m}$ if and only if it includes $C_{n+i}$ for all $i \leq$ $m$. Its intersection with $\left\{L_{i}\right\}$ is therefore the image of an interval in $\mathbb{N}$. Hence $X^{s s}\left(L_{i+1}\right) \backslash X^{s s}\left(L_{i}\right)$ are all disjoint; but each one is open in the complement of $X^{s s}\left(L_{0}\right)$ and the preceding ones. Since varieties are noetherian, this implies there exists $i_{0}$ such that for all $i \geq i_{0}, X^{s s}\left(L_{i+1}\right) \backslash X^{s s}\left(L_{i}\right)=\emptyset$, and hence $X^{s s}\left(L_{i+1}\right) \subset$ $X^{s s}\left(L_{i}\right)$. It therefore suffices to show that there can be no infinite sequence of open proper inclusions, all having good quotients.

Write $U_{i}=X^{s s}\left(L_{i}\right)$, and concentrate first on the single inclusion $U_{i+1} \subset U_{i}$. Since $U_{i} \backslash U_{i+1}$ is closed and nonempty in $U_{i}$, it contains a closed orbit $G \cdot x$, say of dimension $q$. The union of orbits in $U_{i}$ having dimension $q$ or less is a closed set; let $Q$ be an irreducible component intersecting $G \cdot x$. Then $G \cdot Q$ is invariant and closed in $U_{i}$, and $(G \cdot x) / / G\left(L_{i}\right)$ is in $(G \cdot Q) / / G\left(L_{i}\right)$, but not in $\left(G \cdot Q \cap U_{i+1}\right) / / G\left(L_{i}\right)$. Hence $\left(G \cdot Q \cap U_{i+1}\right) / / G\left(L_{i}\right)$ is a proper open subset of the irreducible set $(G \cdot Q) / / G\left(L_{i}\right)$. But it is also a closed subset; indeed, it is the image by the natural projective morphism $X / / G\left(L_{i+1}\right) \rightarrow X / / G\left(L_{i}\right)$ of $\left(G \cdot Q \cap U_{i+1}\right) / / G\left(L_{i+1}\right)$, which is closed by the definition of a good quotient for $L_{i+1}$, since $G \cdot Q \cap U_{i+1}$ is invariant and closed in $U_{i+1}$. Hence $\left(G \cdot Q \cap U_{i+1}\right) / / G\left(L_{i}\right)$ is empty, so $Q \cap U_{i+1}$ itself is empty. There are only finitely many possible values for $q$, and only finitely many components $Q$ for each $q$, so only finitely many inclusions like $U_{i+1} \subset U_{i}$ are possible.

Given that $E_{\mathbb{Q}}^{G}$ is polyhedral in $f_{\mathbb{Q}}^{-1}\left(A_{\mathbb{Q}}\right)$, this result is equivalent to the assertion in [9] that there are only finitely many walls. 


\section{THE GENERAL CASE}

We now embark on our generalization of the results of $\S 1$. So let $G$ be a reductive group over $k$, acting on a quasi-projective variety $X$ over $k$, projective over an affine variety.

All of the arguments in this section use the following trick.

(3.1) Construction. Let $L_{0}, \ldots, L_{m}$ be ample linearizations. Let $\Delta$ be the $m$ simplex $\left\{\left(t_{i}\right) \in \mathbb{Q}^{m+1} \mid \sum t_{i}=1\right\}$. Then for any $t=\left(t_{i}\right) \in \Delta, L(t)=\bigotimes_{i} L_{i}^{t_{i}}$ is a fractional linearization on $X$. We refer to the set $\{L(t) \mid t \in \Delta\}$ as a simplicial family of fractional linearizations.

Put

$$
Y=\mathbb{P}\left(\bigoplus_{i} L_{i}\right)=\operatorname{Proj} \sum_{j_{i} \in \mathbb{N}} H^{0}\left(\bigotimes_{i} L_{i}^{j_{i}}\right)
$$

and let $q: Y \rightarrow X$ be the projection. Then $G$ acts naturally on $\bigoplus_{i} L_{i}$, hence on $Y$ with a canonical linearization on the ample bundle $\mathcal{O}(1)$. Likewise, the $m$-parameter torus $T=\left\{\lambda \in k^{m+1} \mid \prod_{i} \lambda_{i}=1\right\}$ acts on $\bigoplus_{i} L_{i}$ by $\lambda\left(u_{i}\right)=\left(\lambda_{i} u_{i}\right)$, and hence on $Y$. This $T$-action commutes with the $G$-action. Moreover, since it comes from $\bigoplus_{i} L_{i}$, it too is linearized on $\mathcal{O}(1)$. But this obvious linearization is not the only one. Indeed, any $t \in \Delta$ determines a fractional character of $T$ by $t(\lambda)=\prod_{i} \lambda_{i}^{t_{i}}$; then $\lambda\left(u_{i}\right)=\left(t^{-1}(\lambda) \lambda_{i} u_{i}\right)$ determines a fractional linearization depending on $t$. This gives a simplicial family $M(t)$ of fractional linearizations on $\mathcal{O}(1)$ of the $T$-action on $Y$, each compatible with the canonical linearization of the $G$-action. In other words, $M(t)$ is a family of fractional linearizations of the $G \times T$-action on $Y$. Let $Y^{s s}(t)$ be the semistable set for this action and linearization, and let $Y^{s s}(G)$ be the semistable set for the $G$-action alone. For any $t, Y^{s s}(t) \subset Y^{s s}(G)$.

With respect to $M(t), T$ acts trivially on $H^{0}\left(\bigotimes_{i} L_{i}^{j_{i}}\right)$ if and only if $j_{i}=n t_{i}$ for some fixed $n$. Hence the subalgebra of $T$-invariants is $\sum_{n} H^{0}\left(\left(\bigotimes_{i} L_{i}^{t_{i}}\right)^{n}\right)$. The quotient $Y / / T(t)$ is therefore $X$, but with the residual $G$-action linearized by $L(t)$. Consequently $(Y / / T(t)) / / G=X / / G(t)$, the original quotients of interest. Moreover, $X^{s s}(t)=q\left(Y^{s s}(t)\right)$.

Let $Z$ be the quotient $Y / / G$ with respect to the canonical linearization defined above, and let $p: Y^{s s}(G) \rightarrow Z$ be the quotient. Then the $M(t)$ descend to a simplicial family $N(t)$ of fractional linearizations on $\mathcal{O}(1)$ of the residual $T$-action on $Z$, and $Y^{s s}(t)=p^{-1}\left(Z^{s s}(t)\right)$. When two group actions commute, the order of taking the quotient does not matter, so $(Y / / T(t)) / / G=(Y / / G) / / T(t)=Z / / T(t)$. So we have constructed a variety $Z$, acted on by a torus $T$, and a simplicial family $N(t)$ of fractional linearizations on $\mathcal{O}(1)$, such that $Z / / T(t)=X / / G(t)$. Moreover, $X^{s s}(t)=q\left(p^{-1}\left(Z^{s s}(t)\right)\right)$.

As a first application of this construction, let us prove the result asserted in the last section.

Proof of (2.3). It suffices to prove the statements about polyhedrality and semistable sets, as the remaining statements follow from the categorical properties of good quotients. The only exception is the statement about $\mathcal{O}(1)$, which holds simply because the pullback induces a linear injection $\mathrm{NS}_{\mathbb{Q}}(X / / G(t)) \rightarrow \mathrm{NS}_{\mathbb{Q}}^{G}\left(X^{s s}(t)\right)$ taking $\mathcal{O}(1)$ to $L(t)$.

Consider first the case where $X=\mathbb{P}^{n-1}$ and $G$ is an $m$-dimensional torus $T \subset \mathrm{SL}(n)$. This case is treated in [7, 28], but we describe the proof here for 
completeness. Choose coordinates $x_{0}, x_{1}, \ldots, x_{n}$ such that $T$ is a subgroup of the group of all diagonal matrices. For any linearization $L(t)$, the $T$-semistable set is a union of orbits of this larger torus. Since there are only finitely many of these, it suffices to show that the set of linearizations for which a fixed orbit is semistable forms a convex polyhedral cone in $\mathrm{NS}^{G}$. In fact, we will see that they are all images of faces of the standard simplicial cone under a fixed linear map.

Regard $T$ as

$$
\left\{\operatorname{diag} \prod_{i=1}^{m} \lambda_{i}^{r_{i j}} \mid \lambda_{i} \in k^{\times}\right\}
$$

for some integer-valued $m \times n$ matrix $R=\left(r_{i j}\right)$. The corresponding action of $T$ on $\mathcal{O}(1)$ determines a linearization $L$, and by $(2.2)$, any element of $\mathrm{NS}_{\mathbb{Q}}^{T}(X)$ is of the form $L^{p} \otimes t$, where $p \in \mathbb{Q}$ and $t \in \chi(T) \otimes \mathbb{Q}$. But $\chi(T) \cong \mathbb{Z}^{m}$ via $\left(\lambda_{i}\right) \mapsto \prod_{i} \lambda_{i}^{-t_{i}}$ for $t=\left(t_{i}\right) \in \mathbb{Z}^{m}$, so $\chi(T) \otimes \mathbb{Q} \cong \mathbb{Q}^{m}$ and $\mathrm{NS}_{\mathbb{Q}}^{T}(X) \cong \mathbb{Q} \times \mathbb{Q}^{m}$. The ample cone $f_{\mathbb{Q}}^{-1}\left(A_{\mathbb{Q}}\right)$ is of course $\left\{(p, t) \subset \mathbb{Q} \times \mathbb{Q}^{m} \mid p \geq 0\right\}$.

For $q=\left(q_{j}\right) \in \mathbb{Z}^{n}$, the monomial $\prod_{j} x_{j}^{q_{j}}$, regarded as a section of $\mathcal{O}(p)$ where $p=\sum_{i} q_{i}$, is then invariant with respect to the linearization $(p, t)$ if and only if the matrix equation $R q=t$ holds. Because the weight spaces of the $T$-action on $k\left[x_{1}, \ldots, x_{n}\right]$ are spanned by monomials, an orbit is semistable if and only if there is an invariant monomial nonvanishing on that orbit. But for any subset $J \subset\{1, \ldots, n\}$, the monomials nonvanishing on the orbit $\left\{\left(x_{i}\right) \in \mathbb{P}^{n-1} \mid x_{i}=0 \Leftrightarrow\right.$ $j \in J\}$ are exactly those corresponding to $\left\{\left(q_{j}\right) \in \mathbb{Z}^{n} \mid q_{j} \geq 0, q_{j}=0\right.$ if $\left.j \in J\right\}$. So the orbit is semistable with respect to $(p, t)$ if and only if $(p, t) \in\left\{\left(\sum q_{j}, R q\right) \mid q \in\right.$ $\mathbb{Q}^{m}, q_{j} \geq 0, q_{j}=0$ if $\left.j \in J\right\}$. This is the image of a face of the standard simplicial cone in a fixed linear map, as promised.

Now for arbitrary $X$ and $G$, choose a collection of simplices in $f_{\mathbb{Q}}^{-1} A_{\mathbb{Q}} \subset \mathrm{NS}_{\mathbb{Q}}^{G}$ such that every vertex is in $\mathrm{NS}^{G}$, and for every $L \in f_{\mathbb{Q}}^{-1} A_{\mathbb{Q}}$, some fractional power $L^{m}$ is in one of the simplices. The construction (3.1) applies, so for each simplex $\Delta$ there exist $Z \subset \mathbb{P}^{n}$, a simplicial family $N(t)$ in $\mathrm{NS}^{T}\left(\mathbb{P}^{n}\right)$, and maps $p: Y \rightarrow Z$ and $q: Y \rightarrow X$ such that $X^{s s}(t)=q\left(p^{-1}\left(Z^{s s}(t)\right)\right)$ for all $t \in \Delta$. The conclusions of (2.3) are preserved by restriction to a $T$-invariant subvariety, so they hold for $Z$ and $T(t)$. Together with the homogeneity property of quotients mentioned in $\S 2$, this proves the analogue of (2.3) for $X$ and $G(t)$ where $f_{\mathbb{Q}}^{-1} A_{\mathbb{Q}}$ is replaced by the cone over each simplex. This local analogue implies (2.3) itself, except that it only shows $E_{\mathbb{Q}}^{G}$ and the chambers to be polyhedral locally. At this point we may appeal without circularity to (2.4), concluding that if $E_{\mathbb{Q}}^{G}$ has infinitely many walls, then so does some chamber. So to complete the proof, it suffices to show that each chamber has only finitely many walls.

Suppose not. Then there is a chamber $C$ having infinitely many walls $W_{i}$. Each wall corresponds to a different semistable set: this is true for each orbit closure of a torus action on projective space [7,28], so true in general by the construction of (3.1). Since the set $\left\{L: x \in X^{s s}(L)\right\}$ is convex for all $x$ in $X$, for $i$ distinct from $j$, the open sets $X^{s s}\left(W_{i}\right)$ and $X^{s s}\left(W_{j}\right)$ intersect exactly in $X^{s s}(C)$. This is impossible if there are infinitely many $W_{i}$.

The rest of this section and all of $\S \S 4$ and 5 will refer to the following set-up. Let $X$ and $G$ be as before. Let $L_{+}$and $L_{-}$be ample linearizations such that, if 
$L(t)=L_{+}^{t} L_{-}^{1-t}$ for $t \in[-1,1]$, there exists $t_{0} \in(-1,1)$ such that $X^{s s}(t)=X^{s s}(+)$ for $t>t_{0}$ and $X^{s s}(t)=X^{s s}(-)$ for $t<t_{0}$.

For example, (2.3) implies that this is the case if $X$ is normal and projective, $L_{ \pm}$ are in adjacent chambers, and the line segment between them crosses a wall only at $L\left(t_{0}\right)$. Even in the normal projective case, however, there are other possibilities; for example, $L_{ \pm}$could both lie in the same wall, or $L\left(t_{0}\right)$ could lie on the boundary of $E_{\mathbb{Q}}^{G}$. In the future, $L\left(t_{0}\right)$ will be denoted $L_{0}$.

The following lemma shows how to globalize the results of $\$ 1$ within this setup. Suppose $T \cong k^{\times}$acts on $X$, and let $\sigma \in H^{0}\left(X, L_{0}^{n}\right)^{T}$ for some $n$, so that $X_{\sigma} \subset X^{s s}(0)$ is a $T$-invariant affine.

(3.2) Lemma. Suppose $f\left(L_{-}\right)=f\left(L_{+}\right)$. Then (a) $X^{s s}( \pm) \subset X^{s s}(0)$; (b) $X_{\sigma}^{s s}(0)$ $=X_{\sigma} \cap X^{s s}(0)$; and (c) $X_{\sigma}^{s s}( \pm)=X_{\sigma} \cap X^{s s}( \pm)$; so there is a natural commutative diagram

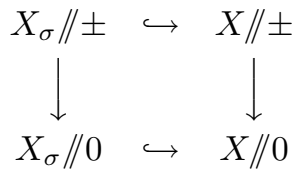

whose rows are embeddings.

Proof. Put $R_{m}=H^{0}\left(X, L_{0}^{m}\right)$, so that $X=\operatorname{Proj} \bigoplus_{m \in \mathbb{N}} R_{m}$, and let $R_{m}=$ $\bigoplus_{n \in \mathbb{Z}} R_{m, n}$ be the weight decomposition for the $k^{\times}$-action. Suppose $x \in X^{s s}(+) \backslash$ $X^{s s}(0)$. Then for $m>0$, every element of $R_{m, 0}$ vanishes at $x$. Since $\bigoplus_{m} R_{m}$ is finitely-generated, this implies that, for $m / n$ large enough, every element of $R_{m, n}$ vanishes at $x$. But then there exists $t>t_{0}$ such that $x \notin X^{s s}(t)$, contradicting the set-up. The proof for $X^{s s}(-)$ is similar. This proves (a).

Without loss of generality suppose $\sigma \in H^{0}\left(X, L_{0}\right)$. Then $\sigma \in R_{1,0}$ and $X_{\sigma}=$ $\operatorname{Spec}\left(\sigma^{-1} R\right)_{0}$. Since $\bigoplus_{m} R_{m, 0}$ is finitely-generated, for $m$ large the map $R_{m, 0} \rightarrow$ $\left(\sigma^{-1} R\right)_{0,0}$ given by dividing by $\sigma^{m}$ is surjective. But $R_{m, 0}=H^{0}\left(X, L_{0}^{m}\right)^{T}$ and $\left(\sigma^{-1} R\right)_{0,0}=H^{0}\left(X_{\sigma}, L_{0}^{m}\right)^{T}$ (the latter since $L_{0}^{m}$ is trivial on $X_{\sigma}$ ), so this implies that $X_{\sigma}^{s s}(0)=X_{\sigma} \cap X^{s s}(0)$, hence that $X_{\sigma} / / 0$ embeds in $X / / 0$. This proves (b).

Without loss of generality take $L_{+}$to be $L_{0}$ twisted by the fractional character $\lambda \mapsto \lambda^{1 / p}$ for $p$ large. Since $R$ is finitely-generated, $R_{m, n} \rightarrow\left(\sigma^{-1} R\right)_{0, n}$ is surjective for $m / n$ large. But for $m=n p, R_{m, n}=H^{0}\left(X, L_{+}^{m}\right)^{T}$ and $\left(\sigma^{-1} R\right)_{0, n}=$ $H^{0}\left(X_{\sigma}, L_{+}^{m}\right)^{T}$, so this implies that $X_{\sigma}^{s s}(+)=X_{\sigma} \cap X^{s s}(+)$, hence that $X_{\sigma} / /+$ embeds in $X / /+$. The case of $L_{-}$is similar. This proves (c).

Hence, in studying $k^{\times}$-quotients where $L_{+} \cong L_{-}$as bundles, we may work locally, using the methods of $\S 1$.

(3.3) Theorem. If $X / / G(+)$ and $X / / G(-)$ are both nonempty, then the morphisms $X / / G( \pm) \rightarrow X / / G(0)$ are proper and birational. If they are both small, then the rational map $X / / G(-) \rightarrow X / / G(+)$ is a flip with respect to $\mathcal{O}(1) \rightarrow X / / G(+)$.

Again, this could be proved directly, by first examining the stable sets to show birationality, then applying Kempf's descent lemma to the linearization $L_{+}$. But again, we will use the trick.

Proof of (3.3). Perform the construction of (3.1) on $L_{+}$and $L_{-}$. This gives a variety $Z$ with an action of $T \cong k^{\times}$and a family $N(t)$ of fractional linearizations with $f_{\mathbb{Q}}(N(t))$ constant such that $Z / / T(t)=X / / G(t)$. The whole statement is local 
over $X / / G(0)$, so by (3.2) it suffices to prove it for affines of the form $Z_{\sigma}$, with the $T$-action and fractional linearizations $N(t)$. But this is the case considered in $\S 1$, so (1.1) and (1.6) complete the proof.

There is a converse to (3.3) analogous to (1.7), which we leave to the reader.

(3.4) Application. For an application, suppose that $X$ is normal and projective. Choose any nonzero $M \in \mathrm{NS}_{\mathbb{Q}}^{G} X$, let $L(t)=L \otimes M^{t}$ and consider the ray $\{L(t) \mid t \geq 0\} \subset \mathrm{NS}_{\mathbb{Q}}^{G}(X)$. By (2.3), the quotient $X / / G(t)$ is empty except for $t$ in some bounded interval $[0, \omega]$, and this interval is partitioned into finitely many subintervals in whose interior $X / / G(t)$ is fixed. But when a critical value $t_{0}$ separating two intervals is crossed, there are morphisms $X / / G\left(t_{ \pm}\right) \rightarrow X / / G\left(t_{0}\right)$, which by (3.3) are birational except possibly at the last critical value $\omega$. Since the fractional polarization on $X / / G(t)$ is the image of $L \otimes M^{-t}$ in the natural descent map $\mathrm{NS}_{\mathbb{Q}}^{G}(X) \rightarrow \mathrm{NS}_{\mathbb{Q}}(X / / G(t))$, the descents of $M$ to $\mathbb{Q}$-Cartier divisor classes on $X / / G( \pm)$ are relatively ample for each morphism $X / / G\left(t_{+}\right) \rightarrow X / / G\left(t_{0}\right)$, and relatively negative for each morphism $X / / G\left(t_{-}\right) \rightarrow X / / G\left(t_{0}\right)$. So suppose that each $X / / G\left(t_{+}\right) \rightarrow X / / G\left(t_{0}\right)$ is small when $X / / G\left(t_{-}\right) \rightarrow X / / G\left(t_{0}\right)$ is small, and that each $X / / G\left(t_{+}\right) \rightarrow X / / G\left(t_{0}\right)$ is an isomorphism when $X / / G\left(t_{-}\right) \rightarrow X / / G\left(t_{0}\right)$ is divisorial. It then follows that the finite sequence of quotients $X / / G(t)$ runs the $M$-minimal model program $[13,(2.26)]$ on $X / / G(L)$, where by abuse of notation $M$ denotes its image in the descent map.

For some $d>0$, the ideal sheaves $\left\langle H^{0}\left(X, L_{ \pm}^{n d}\right)^{G}\right\rangle$ and $\left\langle H^{0}\left(X, L_{ \pm}^{d}\right)^{G}\right\rangle^{n}$ on $X$ are equal for all $n \in \mathbb{N}$. For such a $d$, let $\mathcal{I}^{ \pm}=\left\langle H^{0}\left(X, L_{\mp}^{d}\right)^{G}\right\rangle$ (note the reversal of sign), and let $\mathcal{I}^{ \pm} / / G( \pm)$ be the corresponding sheaves of invariants on $X / / G( \pm)$. Also let $\left(\mathcal{I}^{+}+\mathcal{I}^{-}\right) / / G(0)$ be the sheaf of invariants of the ideal sheaf $\mathcal{I}^{+}+\mathcal{I}^{-}$on $X / / G(0)$.

(3.5) Theorem. Suppose $X / / G(+)$ and $X / / G(-)$ are both nonempty. Then the pullbacks of $\left(\mathcal{I}^{+}+\mathcal{I}^{-}\right) / / G(0)$ by the morphisms $X / / G( \pm) \rightarrow X / / G(0)$ are exactly $\mathcal{I}^{ \pm} / / G( \pm)$, and the blow-ups of $X / / G( \pm)$ at $\mathcal{I}^{ \pm} / / G( \pm)$, and of $X / / G(0)$ at $\left(\mathcal{I}^{+}+\mathcal{I}^{-}\right) / / G(0)$, are all naturally isomorphic to the irreducible component of the fibred product $X / / G(-) \times_{X / / G(0)} X / / G(+)$ dominating $X / / G(0)$.

Proof. Construct a variety $Z$ as in the proof of (3.3). Notice that for $d$ large, since $\mathcal{I}_{X}^{ \pm}=\left\langle H^{0}\left(X, L_{ \pm}^{d}\right)^{G}\right\rangle$ on $X$ and $\mathcal{I}_{Z}^{ \pm}=\left\langle H^{0}\left(Z, N_{ \pm}^{d}\right)^{T}\right\rangle$ on $Z$, the pullbacks of both $\mathcal{I}_{X}^{ \pm}$ and $\mathcal{I}_{Z}^{ \pm}$to $Y$ are $\mathcal{I}_{Y}^{ \pm}=\left\langle H^{0}\left(Y, M_{ \pm}^{d}\right)^{G \times T}\right\rangle$. Hence $\mathcal{I}_{X}^{ \pm}$and $\mathcal{I}_{Z}^{ \pm}$have the same sheaves of invariants on the quotients $Z / / T(t)=X / / G(t)$. It therefore suffices to prove the statement for $Z$ and $N_{ \pm}$. All statements are local over $Z / / T(0)$, so by (3.2) it suffices to prove them for affines of the form $Z_{\sigma}$. But this is the case considered in $\S 1$, so (1.8) and (1.9) complete the proof.

\section{The Smooth Case: Strong Results}

In the next two sections we seek to generalize the other two main results of $\S 1,(1.13)$ and (1.15). Indeed, we will give two different generalizations of each. The generalizations in $\S 4$ make fairly strong hypotheses, and prove that, as in $\S 1$, $X^{ \pm} / / G( \pm)$ are locally trivial over $X^{0} / / G(0)$. Moreover, the proofs are quite easy using the tools already at hand. Those in $\S 5$ relax the hypotheses somewhat, but conclude only that $X^{ \pm} / / G( \pm)$ are locally trivial in the étale topology. The proofs 
therefore require étale covers and are more difficult; in fact we confine ourselves to a sketch of the étale generalization of (1.15).

Let $X, G$, and $L_{ \pm}$be as in $\S 3$. As in (3.1), let $Y=\mathbb{P}\left(L_{+} \oplus L_{-}\right)$, let $T$ be the torus acting on $Y$, let $Z=Y / / G$, and let $p: Y^{s s}(G) \rightarrow Z$ be the quotient morphism. Fix the isomorphism $T \cong k^{\times}$given by projection on the first factor. Define $Y^{ \pm}$, $Y^{0}, Z^{ \pm}$, and $Z^{0}$ similarly to $X^{ \pm}$and $X^{0}$. Also let $i_{ \pm}: X \rightarrow Y$ be the embeddings given by the sections at 0 and $\infty$. Write $q: Y \rightarrow X$ for the projection as before, but let $\pi$ denote the restriction of $q$ to $Y \backslash\left(i_{+}(X) \cup i_{-}(X)\right)$. So in particular $\pi^{-1}(X)$ denotes $Y \backslash\left(i_{+}(X) \cup i_{-}(X)\right)$ itself.

(4.1) Lemma. $X^{s s}( \pm) \subset X^{s s}(0)$.

Proof. This is true for $Z$ by (3.2)(a), but $X^{s s}( \pm)=q\left(p^{-1}\left(Z^{s s}( \pm)\right)\right)$ and $X^{s s}(0)=$ $q\left(p^{-1}\left(Z^{s s}(0)\right)\right)$.

(4.2) Lemma. (a) $i_{ \pm}(X) \cap Y^{s s}(0)=\emptyset$; (b) $i_{ \pm}(X) \cap Y^{s s}(G)=i_{ \pm}\left(X^{s s}( \pm)\right)$; (c) $\pi^{-1}(X) \cap Y^{s s}(0)=\pi^{-1}(X) \cap Y^{s s}(G)=\pi^{-1}\left(X^{s s}(0)\right)$.

Proof. For $i_{ \pm}(x)$ to be in $Y^{s s}(0)$, it must certainly be semistable for the $T$-action on the fiber $q^{-1}(x)=\mathbb{P}^{1}$. But in the fractional linearization $M_{0}, T$ acts with nontrivial weight on both homogeneous coordinates of $\mathbb{P}^{1}$, so any invariant section of $\mathcal{O}(n)$ for $n>0$ must vanish both at 0 and $\infty$. Hence $i_{ \pm}(x)$ are unstable, which proves (a). However, for $i_{ \pm}(x)$ to be in $Y^{s s}(G)$ requires only that the section of $\mathcal{O}(n)$ which is nonzero at $x$ be $G$-invariant. Pushing down by $q$ shows that

$$
H^{0}(Y, \mathcal{O}(n))=H^{0}\left(X, \bigoplus_{j=0}^{n} L_{+}^{j} \otimes L_{-}^{n-j}\right)=\bigoplus_{j=0}^{n} H^{0}\left(X, L_{+}^{j} \otimes L_{-}^{n-j}\right)
$$

and a section of $\mathcal{O}(n)$ is nonzero at $i_{ \pm}(x)$ if and only if its projection on $H^{0}\left(X, L_{ \pm}^{n}\right)$ is nonzero at $x$. Hence $i_{ \pm}(x) \in X^{s s}(G)$ if and only if $x \in X^{s s}( \pm)$, which proves (b).

With respect to the fractional linearization $M(t)$, the $T$-invariant subspace in the above decomposition consists of the summand $H^{0}\left(X, L_{+}^{j} \otimes L_{-}^{n-j}\right)$, if any, such that $L_{+}^{j} \otimes L_{-}^{n-j}$ is a power of $L(t)$, and an invariant section is nonzero on $\pi^{-1}(x)$ if and only if the corresponding element of $H^{0}\left(X, L_{+}^{j} \otimes L_{-}^{n-j}\right)$ is nonzero at $x$. Hence there is a $G$-invariant section of some $\mathcal{O}(n)$ nonvanishing on $\pi^{-1}(x)$ if and only if there is a $G$-invariant section of some $L(t)^{n}$ nonvanishing at $x$; this implies $\pi^{-1}(x) \subset Y^{s s}(G)$ if and only if $x \in \cup_{t} X^{s s}(t)$, which equals $X^{s s}(0)$ by (4.1). On the other hand, $x \in X^{s s}(0)$ if and only if there is a $G$-invariant section of some $L_{0}^{n}$ nonvanishing at $x$, and hence a $G \times T$-invariant section of some $M_{0}^{n}$ nonvanishing on $\pi^{-1}(x)$, that is, $\pi^{-1}(x) \subset Y^{s s}(0)$. This proves (c).

Let $X^{ \pm}$and $X^{0}$ be the intersections with $X^{s s}(0)$ of the supports of the sheaves $\mathcal{I}^{ \pm}$and $\mathcal{I}^{+}+\mathcal{I}^{-}$, defined as in $\S 3$. Note that this generalizes the definitions of $\S 1$. Indeed,

$$
\begin{aligned}
X^{ \pm} & =X^{s s}(0) \backslash X^{s s}(\mp) ; \\
X^{0} & =X^{s s}(0) \backslash\left(X^{s s}(+) \cup X^{s s}(-)\right) .
\end{aligned}
$$

(4.3) Lemma. (a) $\pi^{-1} X^{ \pm}=Y^{ \pm}=p^{-1} Z^{ \pm}$; (b) $\pi^{-1} X^{0}=Y^{0}=p^{-1} Z^{0}$.

Proof. These follow immediately from $\pi^{*} \mathcal{I}_{X}^{ \pm}=\mathcal{I}_{Y}^{ \pm}=p^{*} \mathcal{I}_{Z}^{ \pm}$.

Choose $x \in X^{0}$; throughout this section, we will assume the following. 
(4.4) Hypothesis. Suppose that $X$ is smooth at $x \in X^{0}$, that $G \cdot x$ is closed in $X^{s s}(0)$, and that $G_{x} \cong k^{\times}$.

Note that if $G_{x} \cong k^{\times}$for all $x \in X^{0}$, then an orbit in $X^{0}$ cannot specialize in $X^{0}$, so it is closed in $X^{0}$ and hence in $X^{s s}(0)$. So the second part of the hypothesis is redundant in this case. The third part is necessary, as the counterexample (5.8) will show. But it is always true when $G$ is a torus or when $G$ acts diagonally on the product of its flag variety with another variety [9].

Since $x \in X^{s s}(0), G_{x}$ acts trivially on $\left(L_{0}\right)_{x}$. If it acts nontrivially on $\left(L_{+}\right)_{x}$, requiring it to act with some negative weight $v_{+}<0$ fixes an isomorphism $G_{x} \cong k^{\times}$. It then acts on $\left(L_{-}\right)_{x}$ with some positive weight $v_{-}>0$. To obtain the first, stronger generalizations, assume that these two weights are coprime: $\left(v_{+}, v_{-}\right)=1$. When $X$ is normal and projective and $L_{ \pm}$are in adjacent chambers, this additional hypothesis can be interpreted as follows. The weight of the $G_{x}$-action defines a homomorphism $\rho: \mathrm{NS}^{G} \rightarrow \mathbb{Z}$, and $L_{ \pm}$can be chosen within their chambers to satisfy this hypothesis, and the conditions of the set-up, if and only if $\rho$ is surjective. Again, this is always true when $G$ is a torus or when $G$ acts diagonally on the product of its flag variety with another variety.

If $\left(v_{+}, v_{-}\right)=1$, then $\pi^{-1}(x)$ is contained in a $G$-orbit, so $p\left(\pi^{-1}(x)\right)$ is a single point in $Z$.

(4.5) Lemma. If (4.4) holds and $\left(v_{+}, v_{-}\right)=1$, then $L_{ \pm}$can be chosen so that $G$ acts freely on $Y$ at $\pi^{-1}(x)$ and $Z$ is smooth at $p\left(\pi^{-1}(x)\right)$.

Proof. Since $G_{x}$ acts on $\left(L_{ \pm}\right)_{x}$ with weights $v_{ \pm}$, there exist positive powers of $L_{+}$ and $L_{-}$whose weights add to 1 . Replace $L_{ \pm}$by these powers. Then $G_{x}$ acts freely on $\left(L_{+} \otimes L_{-}^{-1}\right)_{x} \backslash 0$. But this is exactly $\pi^{-1}(x)$, so $G$ acts freely on $Y$ at $\pi^{-1}(x)$. To show that $Z$ is smooth at $p\left(\pi^{-1}(x)\right)$, it therefore suffices to show that $\pi^{-1}(x) \subset Y^{s}(G)$, that is, that the $G$-orbit of $\pi^{-1}(x)$ is closed in $Y^{s s}(G)$. But if $y \in Y^{s s}(G)$ is in the closure of $G \cdot \pi^{-1}(x)$, then $y \notin i_{ \pm}(X)$ by (4.2)(b) and (4.3)(b), so $y \in \pi^{-1}\left(x^{\prime}\right)$ for some $x^{\prime} \in X^{0}$ by (4.2)(c). Then $x^{\prime}$ is in the closure of $G \cdot x \subset X^{0}$, so by (4.4), $x^{\prime} \in G \cdot x$ and hence $y \in G \cdot \pi^{-1}(x)$.

(4.6) Proposition. If (4.4) holds and $\left(v_{+}, v_{-}\right)=1$, then (a) $X^{0}$ is smooth at $x$; (b) on a neighborhood of $x$ in $X^{0}$, there exists a vector bundle $N$ with $k^{\times}$-action, whose fiber at $x$ is naturally isomorphic to $N_{G \cdot x / X}$; (c) the bundle $N^{0}$ of zero weight spaces of $N$ is exactly the image of $T X^{0}$ in $N$; (d) the bundles $N^{ \pm}$of positive and negative weight spaces of $N$ are naturally isomorphic to $N_{X^{0}} / X^{ \pm}$.

Proof. By (1.12)(a), (4.3)(b) and (4.5), $Z^{0}$ is smooth at $p\left(\pi^{-1}(x)\right)$, and $Y^{0}$ is locally a principal $G$-bundle over $Z^{0}$. Hence $Y^{0}$ is smooth at $\pi^{-1}(x)$, so $X^{0}$ is smooth at $x$. The bundle $N_{Z}$ is just $\left.T Z\right|_{Z^{0}}$, so define $N_{Y}=p^{*} N_{Z}$. This is acted upon by $k^{\times}$, so by Kempf's descent lemma [10] descends to a bundle $N_{X}$ which has the desired property. This proves (b); the proofs of (c) and (d) are similar, using (1.12)(c) and (d).

As in $\S 1$, let $w_{i}^{ \pm} \in \mathbb{Z}$ be the weights of the $k^{\times}$-actions on $N^{ \pm}$.

(4.7) Theorem. If (4.4) holds and $\left(v_{+}, v_{-}\right)=1$, then over a neighborhood of $x$ in $X^{0} / / G(0), X^{ \pm} / / G( \pm)$ are locally trivial fibrations with fiber the weighted projective space $\mathbb{P}\left(\left|w_{i}^{ \pm}\right|\right)$.

Proof. This now follows immediately from (1.13) and (4.5). 
If $X / / G( \pm)$ are both nonempty, then $X^{ \pm} / / G( \pm)$ are the supports of the blow-up loci of (3.5). But if $X / / G(-)=\emptyset$, then $X^{+} / / G(+)=X / / G(+)$ and $X^{0} / / G(0)=$ $X / / G(0)$, so (4.7) says the natural morphism $X / / G(+) \rightarrow X / / G(0)$ is a locally trivial weighted projective fibration.

If moreover all $w_{i}^{ \pm}= \pm w$ for some $w$, then for any linearization $L$ such that $L_{x}$ is acted on by $G_{x}$ with weight -1 , the bundles $N^{ \pm} \otimes L^{ \pm w}$ are acted upon trivially by all stabilizers. So by Kempf's descent lemma [10] they descend to vector bundles $W^{ \pm}$over a neighborhood of $x$ in $X^{0} / / G(0)$.

(4.8) Theorem. Suppose that (4.4) holds, that $\left(v_{+}, v_{-}\right)=1$, and that all $w_{i}^{ \pm}=$ $\pm w$ for some $w$. Then over a neighborhood of $x$ in $X^{0} / / G(0), X^{ \pm} / / G( \pm)$ are naturally isomorphic to the projective bundles $\mathbb{P} W^{ \pm}$, their normal bundles are naturally isomorphic to $\pi_{ \pm}^{*} W^{\mp}(-1)$, and the blow-ups of $X / / G( \pm)$ at $X^{ \pm} / / G( \pm)$, and of $X / / G(0)$ at $X^{0} / / G(0)$, are all naturally isomorphic to the fibred product $X / / G(-) \times_{X / / G(0)} X / / G(+)$.

Proof. First notice that, although $W^{ \pm}$depend on the choice of $L$, the projectivizations $\mathbb{P} W^{ \pm}$, and even the line bundle $\mathcal{O}(1,1) \rightarrow \mathbb{P} W^{+} \times_{X^{0} / / G(0)} \mathbb{P} W^{-}$, are independent of $L$. Now on $Z$, taking $L \cong \mathcal{O}$ yields $W^{ \pm}=N^{ \pm}$. But $N_{Y}^{ \pm}=p^{*} N_{Z}^{ \pm}$, so taking $L=p^{*} \mathcal{O}$ on $Y$, with the induced linearization, yields $W_{Y}^{ \pm}=W_{Z}^{ \pm}$. On the other hand, $N_{Y}^{ \pm}=\pi^{*} N_{X}^{ \pm}$also, so for another choice of $L$ on $Y, W_{Y}^{ \pm}=W_{X}^{ \pm}$. Hence $\mathbb{P} W_{X}^{ \pm} \cong \mathbb{P} W_{Z}^{ \pm}$, and the line bundles $\mathcal{O}(1,1) \rightarrow \mathbb{P} W_{X}^{+} \times_{X^{0} / / G(0)} \mathbb{P} W_{X}^{-}$and $\mathcal{O}(1,1) \rightarrow \mathbb{P} W_{Z}^{+} \times{ }_{Z^{0}} \mathbb{P} W_{Z}^{-}$correspond under this isomorphism; pushing down and taking duals, the bundles $\pi_{ \pm}^{*} W_{X}^{\mp}(-1) \rightarrow \mathbb{P} W_{X}^{ \pm}$and $\pi_{ \pm}^{*} W_{Z}^{\mp}(-1) \rightarrow \mathbb{P} W_{Z}^{ \pm}$also correspond. On the other hand, by (4.3)(a) $X^{ \pm} / / G( \pm)=Z^{ \pm} / / T( \pm)$. The theorem then follows from (1.15) together with (3.2).

The hypothesis on $w_{i}^{ \pm}$is most easily verified as follows.

(4.9) Proposition. If every 0-dimensional stabilizer is trivial near $x$, then all $w_{i}^{ \pm}= \pm 1$.

Proof. If not all $w_{i}^{ \pm}= \pm 1$, then by (1.12)(b) there is a point $z \in Z$ with proper nontrivial stabilizer $T_{z}$ such that $p\left(\pi^{-1}(x)\right)$ is in the closure of $T \cdot z$. Then any $y \in p^{-1}(z)$ has nontrivial 0-dimensional stabilizer $(G \times T)_{y}$, and the closure of $(G \times T) \cdot y$ contains $\pi^{-1}(x)$. But then $G_{\pi(y)} \cong(G \times T)_{y}$, and the closure of $G \cdot \pi(y)$ contains $x$.

For most applications, the hypothesis (4.4) will hold for all $x \in X^{0}$. Then the conclusions of (4.7) and (4.8) hold globally, because they are natural. Notice, however, that if $X^{0}$ is not connected, then the $w_{i}^{ \pm}$need be only locally constant.

\section{The SMooth Case: Étale Results}

If $\left(v_{+}, v_{-}\right) \neq 1$, however, then the proof of (4.5) fails, and $X^{ \pm} / / G( \pm)$ need not be locally trivial over $X^{0} / / G(0)$, even if (4.4) holds: see (6.2) for a counterexample. But they will be locally trivial in the étale topology. The proof uses the Luna slice theorem. The first step, however, is to check that $v_{ \pm}$are always nonzero. As always, let $L_{ \pm}$and $L_{0}$ be as in the set-up of $\S 3$.

(5.1) Lemma. If (4.4) holds, then $G_{x}$ acts nontrivially on $\left(L_{ \pm}\right)_{x}$. 
Proof. If $G_{x}$ acts trivially on $L_{+}$(and hence on $L_{-}$), then the embedding $k^{\times}=$ $\pi^{-1}(x) \subset Y$ descends to an embedding $k^{\times} \subset Z$. This is completed by two points, which must come from two equivalence classes of $G$-orbits in $Y^{s s}(G)$. These semistable orbits cannot be $G \cdot\left(i_{ \pm}(x)\right)$, since $i_{ \pm}(x) \notin Y^{s s}(G)$ by (4.2)(a) and the definition of $X^{0}$. Hence our two classes of semistable $G$-orbits must be contained in $\pi^{-1}(\overline{G \cdot x} \backslash G \cdot x)$. By (4.2)(b) and (c) their images in $\pi$ are in $X^{s s}(0)$. But they are also in the closure of $G \cdot x$, which contradicts (4.4).

Again, requiring $G_{x}$ to act on $\left(L_{+}\right)_{x}$ with negative weight $v_{+}<0$ fixes an isomorphism $G_{x} \cong k^{\times}$. It then acts on $\left(L_{-}\right)_{x}$ with positive weight $v_{-}>0$. We no longer require $\left(v_{+}, v_{-}\right)=1$, but assume instead the following.

(5.2) Hypothesis. Suppose that either char $k=0$ or $\left(v_{+}, v_{-}\right)$is coprime to char $k$.

Now choose $y \in \pi^{-1}(x)$, and let $S=(G \times T)_{y}$.

(5.3) Lemma. If (4.4) holds, then there is a fixed isomorphism $S \cong k^{\times}$, and $G_{y}=S \cap G$ is a proper subgroup such that $S / G_{y} \cong T$ naturally. Moreover, if (5.2) holds, then $L_{ \pm}$may be chosen so that $G_{y}$ is reduced.

Proof. Since $\pi$ is the quotient morphism for the $T$-action, $S \subset G_{x} \times T=k^{\times} \times$ $T$; indeed, it is the subgroup acting trivially on $\pi^{-1}(x)$. Since by (5.1) $k^{\times}$acts nontrivially, and $T$ obviously acts with weight 1 , this has a fixed isomorphism to $k^{\times}$, and its intersection with $k^{\times} \times 1$ is a proper subgroup having the desired property.

If char $k=0$, then $G_{y}$ is certainly reduced. Otherwise, let $v=\left(v_{+}, v_{-}\right)$, and replace $L_{+}$and $L_{-}$with positive powers so that $G_{x} \cong k^{\times}$acts on $\pi^{-1}(x)=$ $\left(L_{+} \otimes L_{-}^{-1}\right)_{x}$ with weight $v$. Then $G_{y}=\operatorname{Spec} k[z] /\left\langle z^{v}-1\right\rangle$, which is reduced if $v$ is coprime to char $k$.

Notice that for any $y \in \pi^{-1}(x),(G \times T) \cdot y=G \cdot y$. Together with Lemma (5.3), this implies that any $S$-invariant complement of $T_{y}(G \times T) \cdot y$ in $T_{y} Y$ is also a $G_{y}$-invariant complement of $T_{y}(G \cdot y)$ in $T_{y} Y$. It follows from the definition of the Luna slice $[19,23]$ that a slice for the $(G \times T)$-action at $y$ is also a slice for the $G$-action at $y$. Luna's theorem then implies that there exists a smooth affine $U \subset Y$ containing $y$ and preserved by $S$, and a natural diagram

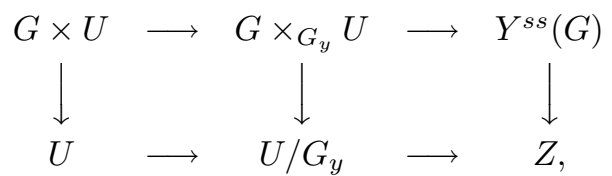

such that the two horizontal arrows on the right are strongly étale with respect to the actions of

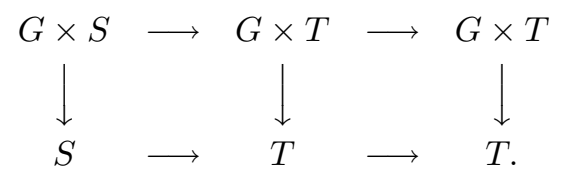

These actions are obvious in every case except perhaps on $G \times U$; there the $G \times S$-action is given by $(g, s) \cdot(h, u)=\left(g h \hat{s}^{-1}, s u\right)$, where $\hat{s}$ is the image of $s$ in the projection $S \rightarrow G$. Each of these actions has a 1-parameter family of fractional linearizations, pulled back from the right-hand column. For any object $V$ in the diagram, define $V^{0}$ and $V^{ \pm}$with respect to these linearizations. 
(5.4) Lemma. For every arrow $f: V \rightarrow W$ in the diagram, $f^{-1}\left(W^{ \pm}\right)=V^{ \pm}$and $f^{-1}\left(W^{0}\right)=V^{0}$.

Proof. This is straightforward for the vertical arrows, and for the morphism $U \rightarrow$ $U / G_{y}$, because they are all quotients by subgroups of the groups which act. The result for $G \times U \longrightarrow G \times \times_{G_{y}} U$ follows from the commutativity of the diagram. As for the strongly étale morphisms, these are treated as in the proof of (1.12)(b).

With this construction, (4.6) can now be strengthened.

(5.5) Proposition. If (4.4) and (5.2) hold, then the conclusions of (4.6) follow even if $\left(v_{+}, v_{-}\right) \neq 1$.

Proof. By (5.3) and (5.4), in a neighborhood of $\pi^{-1}(x)$ in $Y$, a point is in $Y^{0}$ if and only if it has stabilizer conjugate to $S$. Hence in a neighborhood of $x$ in $X$, a point is in $X^{0}$ if and only if it has stabilizer conjugate to $G_{x}$. So if $W \subset X^{0}$ is the fixed-point set for the $G_{x}$-action on a neighborhood of $x \in X$, then a neighborhood of $x$ in $X^{0}$ is precisely the affine quotient of $W \times G$ by the diagonal action of the normalizer of $G_{x}$ in $G$. In particular, this is smooth as claimed in (a), because $W$ is smooth by (1.12)(a) and the normalizer acts freely. Also, its tangent bundle has a natural subbundle consisting of the tangent spaces to the $G$-orbits. Let $N_{X}$ be the quotient of $\left.T X\right|_{X^{0}}$ by this subbundle. Then $N_{X}$ certainly satisfies (b), and $N_{Y}=\pi^{*} N_{X}$, so it suffices to prove (c) and (d) for $Y$ with its $G \times T$-action. But (c) and (d) hold for $U$ with its $S$-action by (1.12)(c) and (d), hence for $G \times U$ with its $G \times S$-action since $N_{U}$ pulls back to $N_{G \times U}$. But the morphism $G \times_{G_{y}} U \rightarrow Y$ is étale, and so is the morphism $G \times U \rightarrow G \times{ }_{G_{y}} U$, since $G_{y}$ is reduced. The result for $Y$ then follows from (5.4), since étale morphisms are isomorphisms on tangent spaces.

Let $w_{i}^{ \pm} \in \mathbb{Z}$ be the weights of the $k^{\times}$-action on $N^{ \pm}$.

(5.6) Theorem. If (4.4) and (5.2) hold, then over a neighborhood of $x$ in $X^{0} / / G(0)$, $X^{ \pm} / / G( \pm)$ are fibrations, locally trivial in the étale topology, with fiber the weighted projective space $\mathbb{P}\left(\left|w_{i}^{ \pm}\right|\right)$.

As before, if $X / / G( \pm)$ are both nonempty, then $X^{ \pm} / / G( \pm)$ are the supports of the blow-up loci of (3.5); but if $X / / G(-)=\emptyset$, then (5.6) says the natural morphism $X / / G(+) \rightarrow X / / G(0)$ is a weighted projective fibration, locally trivial in the étale topology.

The proof requires the following lemma.

(5.7) Lemma. If $\phi: V \rightarrow W$ is a strongly étale morphism of affine varieties with $k^{\times}$-action, then $\phi / / \pm: V / / \pm \rightarrow W / / \pm$ are étale, and $V / / \pm=W / / \pm \times_{W / / 0} V / / 0$.

Proof. Say $V=\operatorname{Spec} R, W=\operatorname{Spec} S$. The $k^{\times}$-actions induce $\mathbb{Z}$-gradings on $R$ and $S$, and $V=W \times_{W / / 0} V / / 0$ implies $R=S \otimes_{S_{0}} R_{0}$. Hence

$$
\bigoplus_{i \in \mathbb{N}} R_{ \pm i}=\bigoplus_{i \in \mathbb{N}} S_{ \pm i} \otimes_{S_{0}} R_{0}
$$

which implies the second statement. Then $\phi / / \pm$ are certainly étale, since being étale is preserved by base change.

Proof of (5.6). The Luna slice $U$ associated to any $y \in \pi^{-1}(x)$ is smooth, and for $S=(G \times T)_{y}, U / / S(t)=\left(U / G_{y}\right) / / T(t)$ since $T=S / G_{y}$ by (5.3). But as stated when 
$U$ was constructed, $U / G_{y}$ is strongly étale over $Z$, so by (5.7) and (3.2) $U / / S(t)$ is étale over $Z / / T(t)=X / / G(t)$, and $U / / S( \pm)=X / / G( \pm) \times_{X / / G(0)} U / / S(0)$. In particular, $U^{0} / / S( \pm)=X^{0} / / G( \pm) \times_{X^{0} / / G(0)} U^{0} / / S(0)$, which is exactly the pullback of $X^{0} / / G( \pm)$ by the étale morphism $U^{0} / / S(0) \rightarrow X^{0} / / G(0)$. The theorem therefore follows from the analogous result (1.13) for quotients of smooth affines by $k^{\times}$.

(5.8) Counterexample. To show that the hypothesis $G_{x} \cong k^{\times}$is necessary in (5.6).

Let $G$ be any semisimple reductive group, and let $V_{+}$and $V_{-}$be representations of $G$. Let $X=\mathbb{P}\left(V_{+} \oplus V_{-} \oplus k\right)$, and let $G \times k^{\times}$act on $X, G$ in the obvious way, and $k^{\times}$ with weights $1,-1,0$. Then $\mathrm{NS}_{\mathbb{Q}}^{G \times k^{\times}} \cong \mathbb{Q}$, with two chambers separated by a wall at 0 . Moreover $X^{ \pm}=\mathbb{P}\left(V_{ \pm} \oplus k\right)$, so $X^{0}=\{(0,0,1)\}$. But $G_{(0,0,1)}=G \times k^{\times}$, so the hypothesis is violated. Now $X^{ \pm} / / k^{\times}( \pm)=\mathbb{P}\left(V_{ \pm}\right)$, so $X^{ \pm} / /\left(G \times k^{\times}\right)( \pm)=\mathbb{P}\left(V_{ \pm}\right) / / G$. This certainly need not be a projective space, as the theorem would predict; see for example the discussion of the case $G=\operatorname{PSL}(2)$ in $\S 6$.

Since $G$ acts on $N^{ \pm}$, there are quotients $N^{ \pm} / / G( \pm)$, which are fibrations with fiber $\mathbb{P}\left(\left|w_{i}^{ \pm}\right|\right)$over a neighborhood of $x$ in $X^{0} / / G(0)$, locally trivial in the étale topology. Notice that by (5.4), since $N_{V}^{ \pm}=N_{V^{0} / V^{ \pm}}$for $V=X, Y, G \times_{G_{y}} U$, and $U$,

$$
\begin{aligned}
N_{U}^{ \pm} / / S( \pm) & =N_{G \times U}^{ \pm} / /(G \times S)( \pm) \\
& =N_{Y}^{ \pm} / /(G \times T)( \pm) \times_{Y^{0}} / /(G \times T)(0) \\
& =N_{X}^{ \pm} / / G( \pm) \times_{X^{0} / / G(0)} U^{0} / / S(0),
\end{aligned}
$$

which is the pullback of $N_{X}^{ \pm} / / G( \pm)$ by the étale morphism $U^{0} / / S(0) \rightarrow X^{0} / / G(0)$. The following result then ought to be true, but proving it conclusively is rather cumbersome, so we content ourselves with a sketch.

(5.9) Theorem. Suppose that (4.4) and (5.2) hold, and that all $w_{i}^{ \pm}= \pm w$ for some $w$. Then $X^{ \pm} / / G( \pm)$ are naturally isomorphic to $N^{ \pm} / / G( \pm)$, and the blow-ups of $X / / G( \pm)$ at $X^{ \pm} / / G( \pm)$, and of $X / / G(0)$ at $X^{0} / / G(0)$, are all naturally isomorphic to the fibred product $X / / G(-) \times_{X / / G(0)} X / / G(+)$.

Sketch of proof. All the blow-ups and the fibred product are empty if either $X / / G(+)$ or $X / / G(-)$ is empty, so suppose they are not. Now $X^{ \pm} / / G( \pm) \rightarrow X^{0} / / G(0)$ are covered in the étale topology by $U^{ \pm} / / S( \pm) \rightarrow U^{0} / / S(0)$ by $(5.7)$, and $N_{X}^{ \pm} / / G( \pm) \rightarrow$ $X^{0} / / G(0)$ are covered in the étale topology by $N_{U}^{ \pm} / / S( \pm) \rightarrow U^{0} / / S(0)$ by the remarks above. But the analogous result for $U$ holds by (1.15). The theorem would therefore follow if we could display a morphism $N_{X}^{ \pm} / / G( \pm) \rightarrow X^{ \pm} / / G( \pm)$ compatible with the étale morphisms and the isomorphisms of (1.15). Unfortunately, this is somewhat awkward to construct. One way to do it is to imitate the argument of (4.8), using a bundle of tangent cones with $k^{\times}$-action over $Z^{0}$, which is typically in the singular locus of $Z$. This requires generalizing the Bialynicki-Birula decomposition theorem to the mildly singular space $Z$, which can still be accomplished using the Luna slice theorem.

The hypothesis on $w_{i}^{ \pm}$is again most easily verified as follows.

(5.10) Proposition. If every 0-dimensional stabilizer is trivial near $x$, then all $w_{i}^{ \pm}= \pm 1$. 
Proof. If not all $w_{i}^{ \pm}= \pm 1$, then for each Luna slice $U$ there is a point $u \in U$ with nontrivial proper stabilizer $S_{u}$. Then any $(g, u) \in G \times U$ satisfies $(G \times S)_{(g, u)} \cong S_{u}$. Since the morphism $G \times U \rightarrow Y$ is étale, this implies that there exists $y \in Y$ with a nontrivial 0-dimensional stabilizer $(G \times T)_{y}$. But then $G_{\pi(y)}=(G \times T)_{y}$.

Again, for most applications, the hypothesis (4.4), and hence the conclusions of (5.6) and (5.9), will hold globally.

\section{THE FIRST EXAMPLE}

In this section we turn to a simple application of our main results, the muchstudied diagonal action of $\operatorname{PSL}(2)$ on the $n$-fold product $\left(\mathbb{P}^{1}\right)^{n}$. This has $n$ independent line bundles, so it is tempting to study the quotient with respect to an arbitrary $\mathcal{O}\left(t_{1}, \ldots, t_{n}\right)$. We will take a different approach, however: to add an $n+1$ th copy of $\mathbb{P}^{1}$, and consider only fractional linearizations on $\left(\mathbb{P}^{1}\right)^{n+1}$ of the form $\mathcal{O}(t, 1,1, \ldots, 1)$. This has the advantage that it does not break the symmetry among the $n$ factors. In other words, the symmetric group $S_{n}$ acts compatibly on everything, so in addition to $\left(\mathbb{P}^{1}\right)^{n}$, we learn about quotients by $\operatorname{PSL}(2)$ of the symmetric product $\left(\mathbb{P}^{1}\right)^{n} / S_{n}=\mathbb{P}^{n}$.

So for any $n>2$, let $\left(\mathbb{P}^{1}\right)^{n}$ be acted on diagonally by $G=\operatorname{PSL}(2)$, fractionally linearized on $\mathcal{O}(1,1, \ldots, 1)$. We wish to study the quotient $\left(\mathbb{P}^{1}\right)^{n} / / G$. The stability condition for this action is worked out in $[24,4.16$; GIT, Ch. 3], using the numerical criterion. This is readily generalized to an arbitrary linearization on $X=\mathbb{P}^{1} \times$ $\left(\mathbb{P}^{1}\right)^{n}=\left(\mathbb{P}^{1}\right)^{n+1}$; indeed for the fractional linearization $\mathcal{O}\left(t_{0}, \ldots, t_{n}\right)$, it turns out that $\left(x_{j}\right) \in X^{s s}\left(t_{j}\right)$ if and only if, for all $x \in \mathbb{P}^{1}$,

$$
\sum_{j=0}^{n} t_{j} \delta\left(x, x_{j}\right) \leq \sum_{j=0}^{n} t_{j} / 2 .
$$

Moreover, $\left(x_{j}\right) \in X^{s}\left(t_{j}\right)$ if and only if the inequality is always strict. We will study the case where $t_{0}$ is arbitrary, but $t_{j}=1$ for $j>0$.

For $t_{0}<1$, it is easy to see that $\mathbb{P}^{1} \times\left(\left(\mathbb{P}^{1}\right)^{n}\right)^{s} \subset X^{s s} \subset \mathbb{P}^{1} \times\left(\left(\mathbb{P}^{1}\right)^{n}\right)^{s s}$. So the projection $X \rightarrow\left(\mathbb{P}^{1}\right)^{n}$ induces a morphism $X / / G\left(t_{0}\right) \rightarrow\left(\mathbb{P}^{1}\right)^{n} / / G$ whose fiber over each stable point is $\mathbb{P}^{1}$. Indeed, each diagonal in $X=\mathbb{P}^{1} \times\left(\mathbb{P}^{1}\right)^{n}$ is fixed by $G$, so descends to $X / / G\left(t_{0}\right)$. Hence, over the stable set in $\left(\mathbb{P}^{1}\right)^{n} / / G, X / / G\left(t_{0}\right)$ is exactly the total space of the universal family.

Now because $G=\operatorname{PSL}(2)$, not $\operatorname{SL}(2)$, the bundle $\mathcal{O}(1,0,0, \ldots, 0)$ has no bona fide linearization, only a fractional one. However, $\mathcal{O}(1,1,0, \ldots, 0)$ does admit a bona fide linearization, as does $\mathcal{O}(1,1, \ldots, 1)$ if $n$ is odd. So these bundles descend to $X / / G\left(t_{0}\right)$ for $t_{0}<1$, yielding line bundles whose restriction to each $\mathbb{P}^{1}$ fiber is $\mathcal{O}(1)$. This implies that, over the stable set in $\left(\mathbb{P}^{1}\right)^{n} / / G, X / / G\left(t_{0}\right)$ is a locally trivial fibration. In particular, if $n$ is odd, it is a fibration everywhere. However, if $n$ is even, there is no $S_{n}$-invariant line bundle having the desired property. Hence the quotient $\left(\mathbb{P}^{1} \times \mathbb{P}^{n}\right) / / G\left(t_{0}\right)=\left(X / / G\left(t_{0}\right)\right) / S_{n}$, though it has generic fiber $\mathbb{P}^{1}$ over $\mathbb{P}^{n} / / G$, and is generically trivial in the étale topology, is not even locally trivial anywhere. It is (generically) what is sometimes called a conic bundle.

To apply our results to this situation, note first that for numerical reasons the stability condition only changes when equality can occur in the inequality above, that is, when $t_{0}=n-2 m$ for some integer $m \leq n / 2$. These will be our walls. A point $\left(x_{j}\right) \in X$ is in $X^{0}$ for one of these walls if it is semistable for $t=t_{0}$, but 
unstable otherwise. This means there exist points $x, x^{\prime} \in \mathbb{P}^{1}$ such that

$$
t \delta\left(x, x_{0}\right)+\sum_{j=1}^{n} \delta\left(x, x_{j}\right) \geq \frac{t+n}{2}
$$

for $t \leq t_{0}$, and

$$
t \delta\left(x^{\prime}, x_{0}\right)+\sum_{j=1}^{n} \delta\left(x^{\prime}, x_{j}\right) \geq \frac{t+n}{2}
$$

for $t \geq t_{0}$, with equality in both if and only if $t=t_{0}$. This requires that all $x_{j}$ be either $x$ or $x^{\prime}$; indeed, $x_{0}$ and exactly $m$ other $x_{j}$ must be $x$, and the $n-m$ remaining $x_{j}$ must be $x^{\prime}$. In particular, this implies $m \geq 0$, so there are only finitely many walls, as expected. On the other hand, any $\left(x_{j}\right) \in X$ of this form will belong to $X^{s s}\left(t_{0}\right)$, provided that $x \neq x^{\prime}$. So $X^{0}\left(t_{0}\right)$ consists of $\left(\begin{array}{l}n \\ m\end{array}\right)$ copies of $\left(\mathbb{P}^{1} \times \mathbb{P}^{1}\right) \backslash \Delta$.

Hence every point $\left(x_{j}\right) \in X^{0}\left(t_{0}\right)$ is stabilized by the subgroup of $G$ fixing $x$ and $x^{\prime}$, which is isomorphic to $k^{\times}$. So the hypothesis (4.4) is satisfied. Moreover, the bundle $\mathcal{O}(2,0,0, \ldots, 0)$ is acted on by this $k^{\times}$with weight 1 , so the strong results of $\S 4$ will apply. Finally, we claim that, even though $X^{0}$ is disconnected, the weights $w_{i}^{ \pm}$are globally constant, and are all \pm 1 . Indeed, it is easy to see that the $w_{i}^{ \pm}$are independent of the component, because the action of the symmetric group $S_{n}$ on $X$ commutes with the $G$-action, and acts transitively on the components of $X^{0}$. To evaluate $w_{i}^{ \pm}$, note that each component is a single orbit, and that setting $x=0, x^{\prime}=\infty$ determines a unique point in this orbit with stabilizer $\left\{\operatorname{diag}\left(\lambda^{-1}, \lambda\right) \mid \lambda \in k^{\times}\right\} / \pm 1$. This acts on $T \mathbb{P}^{1}$ with weight -1 at 0,1 at $\infty$; so it acts on $T_{\left(x_{j}\right)} X$ with $m+1$ weights equal to -1 and $n-m$ weights equal to 1 . But it acts on the $G$-orbit $G / k^{\times}$with one weight equal to 1 and one equal to -1 , so $N$ is acted on with $m$ weights -1 and $n-m-1$ weights 1 . So the very strongest result (4.8) applies. Hence $X^{ \pm} / / G( \pm)$ are bundles with fiber $\mathbb{P}^{n-m-2}$ and $\mathbb{P}^{m-1}$, respectively, over $X^{0} / / G(0)$. Since this is just $\left(\begin{array}{l}n \\ m\end{array}\right)$ points, $X^{ \pm} / / G( \pm)$ are disjoint unions of $\left(\begin{array}{l}n \\ m\end{array}\right)$ projective spaces. For example, when $m=0, X^{+} / / G(+)=X / / G(n-1)=\mathbb{P}^{n-2}$, and when $m=1, X^{-} / / G(-)$ consists of $n$ points. Using the numerical criterion and the tautological family over $X / / G(n-1)$, it is not hard to check that these $n$ points are in general position, and that for general $m, X^{-} / / G(-)$ is the proper transform of the union of all the $m-1$-planes through $m$ of the $n$ points (cf. $\S 8$ of [27]). Thus (4.8), by asserting that the blow-ups of $X / / G( \pm)$ at $X^{ \pm} / / G( \pm)$ are the same, gives a practical way to construct $X / / G(t)$ for any $t$ by blowing up and down the proper transforms of these planes.

In particular, of course, $X / / G(1)=\left(\mathbb{P}^{1}\right)^{n+1} / / G$, so this gives an explicit construction of $\left(\mathbb{P}^{1}\right)^{n+1} / / G$, except that 1 is on a wall for $n+1$ even, so the last step in that case is a contraction rather than a blow-down. We prefer, however, to continue on to the region $t<1$. In this case $X / / G(t)$ is (at least generically) a $\mathbb{P}^{1}$-bundle over $\left(\mathbb{P}^{1}\right)^{n} / / G$. Moreover, the bundle structure, and the whole chain of flips leading up to it, are acted on compatibly by the symmetric group $S_{n}$. As mentioned above, this is an advantage for studying quotients of $\mathbb{P}^{n}$ by $G$. To illustrate this, let us calculate the Betti numbers of $\left(\mathbb{P}^{1}\right)^{n} / / G$ and $\mathbb{P}^{n} / / G$ for $n$ odd, in the case where the ground field is the complex numbers $\mathbb{C}$. These formulas are originally due to Kirwan [18]. 
(6.1) Proposition. For $n$ odd,

$$
P_{t}\left(\left(\mathbb{P}^{1}\right)^{n} / / G\right)=\sum_{m=0}^{(n-1) / 2}\left(\begin{array}{l}
n \\
m
\end{array}\right) \frac{t^{2 m}-t^{2(n-m-1)}}{1-t^{4}}
$$

and

$$
P_{t}\left(\mathbb{P}^{n} / / G\right)=\sum_{m=0}^{(n-1) / 2} \frac{t^{2 m}-t^{2(n-m-1)}}{1-t^{4}} .
$$

Proof. Let $t_{0}=n-2 m$, and $t_{ \pm}=t_{0} \mp 1$. Then the blow-ups of $X / / G( \pm)$ at $X^{ \pm} / / G( \pm)$ are equal. So by the standard formula for Poincaré polynomials of blow-ups,

$$
\begin{aligned}
& P_{t}(X / / G(-))-P_{t}\left(X^{-} / / G(-)\right)+P_{t}(E) \\
& \quad=P_{t}(X / / G(+))-P_{t}\left(X^{+} / / G(+)\right)+P_{t}(E),
\end{aligned}
$$

where $E$ is the exceptional divisor. Cancelling and rearranging yields

$$
P_{t}(X / / G(+))-P_{t}(X / / G(-))=P_{t}\left(X^{+} / / G(+)\right)-P_{t}\left(X^{-} / / G(-)\right) .
$$

But $X^{ \pm} / / G( \pm)$ are $\left(\begin{array}{c}n \\ m\end{array}\right)$ copies of $\mathbb{P}^{n-m-2}$ and $\mathbb{P}^{m-1}$ respectively, so

$$
\begin{aligned}
P_{t}(X / / G(+))-P_{t}(X / / G(-)) & =\left(\begin{array}{c}
n \\
m
\end{array}\right)\left(\frac{1-t^{2(n-m-1)}}{1-t^{2}}-\frac{1-t^{2 m}}{1-t^{2}}\right) \\
& =\left(\begin{array}{c}
n \\
m
\end{array}\right) \frac{t^{2 m}-t^{2(n-m-1)}}{1-t^{2}} .
\end{aligned}
$$

Summed over $m$, the left-hand side telescopes, so for $t<1$

$$
P_{t}(X / / G(t))=\sum_{m=0}^{(n-1) / 2}\left(\begin{array}{l}
n \\
m
\end{array}\right) \frac{t^{2 m}-t^{2(n-m-1)}}{1-t^{2}} .
$$

But for $n$ odd, this is a $\mathbb{P}^{1}$-bundle over $\left(\mathbb{P}^{1}\right)^{n} / / G$, and the Poincaré polynomial of any projective bundle splits, so

$$
P_{t}\left(\left(\mathbb{P}^{1}\right)^{n} / / G\right)=\sum_{m=0}^{(n-1) / 2}\left(\begin{array}{l}
n \\
m
\end{array}\right) \frac{t^{2 m}-t^{2(n-m-1)}}{1-t^{4}},
$$

as desired.

As for $\mathbb{P}^{n} / / G$, it is the quotient of $\left(\mathbb{P}^{1}\right)^{n} / / G$ by the action of the symmetric group $S_{n}$. A result from Grothendieck's Tôhoku paper then implies $[14,20]$ that $H^{*}\left(\mathbb{P}^{n} / / G, \mathbb{C}\right)$ is the $S_{n}$-invariant part of $H^{*}\left(\left(\mathbb{P}^{1}\right)^{n} / / G, \mathbb{C}\right)$. But since $S_{n}$ acts on $X / / G(t)$ for all $t$, the calculation above actually decomposes $H^{*}\left(\left(\mathbb{P}^{1}\right)^{n} / / G, \mathbb{C}\right)$ as a representation of $S_{n}$ : the term with coefficient $\left(\begin{array}{c}n \\ m\end{array}\right)$ gives the multiplicity of the permutation representation induced by the natural action of $S_{n}$ on subsets of $\{1, \ldots, n\}$ of size $m$. The trivial summand of this representation is exactly one-dimensional, so the cohomology of $\mathbb{P}^{n} / / G$ is as stated.

We round off this section by using some of the ideas discussed above to give the counterexample promised in $\S 5$.

(6.2) Counterexample. To show that the hypothesis $\left(v_{+}, v_{-}\right)=1$ is necessary in $(4.7)$. 
Let $V$ be the standard representation of GL(2), and let $W=S^{n} V \otimes\left(\Lambda^{2} V\right)^{-n / 2}$, where $S^{n} V$ is the $n$th symmetric power for some even $n>2$. Let $X=$ $\mathbb{P}\left(V \oplus V^{*} \oplus W\right)$, and let $\mathrm{GL}(2)$ act on $X$. Then $\mathrm{NS}_{\mathbb{Q}}^{\mathrm{GL}(2)} \cong \mathbb{Q}$, with two chambers separated by a wall at 0 . The central $k^{\times} \subset \operatorname{GL}(2) \operatorname{acts}$ on $V, V^{*}$, and $W$ with weight $1,-1$, and 0 respectively, so $X^{+}$is open in $\mathbb{P}(V \oplus W), X^{-}$is open in $\mathbb{P}\left(V^{*} \oplus W\right)$, and $X^{0}$ is open in $\mathbb{P} W$. By construction, the scalars $k^{\times} \subset \mathrm{GL}(2)$ act trivially on $\mathbb{P} W$ with the linearization $L_{0}$, so the action reduces to the action of $\operatorname{PSL}(2)$ on $\mathbb{P}^{n}$ considered above. A generic $x \in \mathbb{P}^{n}$ is stable and is acted on freely by $\operatorname{PSL}(2)$, so $\operatorname{GL}(2)_{x}=k^{\times}$. Moreover, it is stable, so $\operatorname{GL}(2) \cdot x=\operatorname{PSL}(2) \cdot x$ is closed in $\left(\mathbb{P}^{n}\right)^{s s}$ and hence in $X^{s s}(0)$. Therefore (4.4) holds for the GL(2)-action at $x$. On the other hand, the hypothesis $\left(v_{+}, v_{-}\right)=1$ cannot be satisfied: the tautological linearization $L_{0}$ on $\mathcal{O}(1)$ is acted on with weight 0 , and the linearization $L_{+}$obtained by tensoring $L_{0}$ with the character det : GL(2) $\rightarrow k^{\times}$is acted on with weight 2 , but together these generate $\operatorname{Pic}{ }^{\mathrm{GL}(2)} X$.

Now $X^{+} / / k^{\times}(+)=\mathbb{P} V \times \mathbb{P} W$. As a variety with PSL(2)-action, this is exactly $\mathbb{P}^{1} \times \mathbb{P}^{n}$ as considered above. The + linearization on $\mathbb{P} V \times \mathbb{P} W$ corresponds to the linearization given by $t<1$ on $\mathbb{P}^{1} \times \mathbb{P}^{n}$, so $X^{ \pm} / / \mathrm{GL}(2)(+)=(\mathbb{P} V \times \mathbb{P} W) / / \operatorname{PSL}(2)(+)=$ $\left(\mathbb{P}^{1} \times \mathbb{P}^{n}\right) / / \operatorname{PSL}(2)(t)$. As mentioned above, this is a conic bundle, so it is not even locally trivial over $X^{0} / / \mathrm{GL}(2)(0)=\mathbb{P} W / / \operatorname{PSL}(2)(0)=\mathbb{P}^{n} / / \operatorname{PSL}(2)$ at $x$.

\section{Parabolic bundles}

In the last two sections we apply our main results to moduli problems of vector bundles with additional structure over a curve. Throughout these sections, $C$ will denote a smooth projective curve over $k$ of genus $g$.

Fix a point $p \in C$. A parabolic bundle over $C$ with parabolic structure at $p$ is a vector bundle $E$ together with a flag $0=V_{0} \subset V_{1} \subset V_{2} \subset \cdots \subset V_{r}=E_{p}$ in the fiber of $E$ at $p$, labeled with rational weights $0 \leq \ell_{1}<\ell_{2}<\cdots<\ell_{r}<1$. For simplicity we will study only bundles with full flags, so that rank $E=r$. The space of all possible weights is then parametrized by

$$
W=\left\{\left(\ell_{j}\right) \in \mathbb{Q}^{r} \mid 0 \leq \ell_{1}<\ell_{2}<\cdots<\ell_{r}<1\right\} .
$$

A parabolic bundle of rank $r$ and degree $d$ is said to be semistable with respect to $\left(\ell_{j}\right)$ if for all subbundles $F \subset E$ of rank $r^{\prime}$ and degree $d^{\prime}$,

$$
\frac{d^{\prime}+\sum_{i=1}^{r^{\prime}} \ell_{j_{i}}}{r^{\prime}} \leq \frac{d+\sum_{j=1}^{r} \ell_{j}}{r}
$$

where $j_{i}$ run over those integers such that $\operatorname{dim} V_{i} \cap F_{p}-\operatorname{dim} V_{i-1} \cap F_{p}=1$. We refer to $[21,26]$ for basic definitions and results on parabolic bundles.

There are several constructions of the moduli space $M\left(\ell_{j}\right)$ of parabolic bundles semistable with respect to $\left(\ell_{j}\right)$. The one best suited for our purposes is due to Bhosle [3], following Gieseker [12]; so we first review his construction, then hers.

Suppose without loss of generality that $d>>0$, and let $\chi=d+r(1-g)$. Let Quot be the Grothendieck Quot scheme [15] parametrizing quotients $\phi: \mathcal{O}_{C}^{\chi} \rightarrow E$, where $E$ has Hilbert polynomial $\chi+r i$ in $i$, and let $\mathcal{O}^{\chi} \rightarrow \mathbf{E}$ be the universal quotient over Quot $\times C$. Let $R \subset$ Quot be the smooth open subvariety consisting of locally free sheaves $E$ such that $H^{0}\left(\mathcal{O}^{\chi}\right) \rightarrow H^{0}(E)$ is an isomorphism, and let $R^{s s}$ be the subset corresponding to semistable bundles. For $d$ large, every semistable bundle of rank $r$ and degree $d$ is represented by a point in $R$. Let $Z$ be the bundle over $\operatorname{Pic}^{d} C$, constructed as a direct image, with fiber $\mathbb{P} \operatorname{Hom}\left(H^{0}\left(\Lambda^{r} \mathcal{O}^{\chi}\right), H^{0}(M)\right)$ 
at $M$. The group $G=\operatorname{SL}(\chi)$ acts on $R$ and $Z$, and there is a natural $G$-morphism $T: R \rightarrow Z$, and a linearization $L$ on $Z$, such that $T^{-1} Z^{s s}(L)=R^{s s}$. Moreover, the restriction $T: R^{s s} \rightarrow Z^{s s}(L)$ is finite. The existence of a good quotient of $R^{s s}$ by $G$ then follows from a lemma [12, Lemma 4.6] which states that if a set has a good $G$-quotient, then so does its preimage by a finite $G$-morphism. This quotient is the moduli space of semistable bundles on $C$.

To construct the moduli space of semistable parabolic bundles in an analogous way, let $\tilde{R}$ be the bundle $\left.\mathrm{Fl} \mathbf{E}\right|_{R \times\{p\}}$ of full flags in $\mathbf{E}_{p}$. This parametrizes a family of quasi-parabolic bundles; for $d$ large, any bundle which is semistable for some weights $\left(\ell_{j}\right)$ is represented by a point in $\tilde{R}$. Let $\tilde{R}^{s s}\left(\ell_{j}\right)$ be the subset corresponding to parabolic bundles semistable with respect to $\left(\ell_{j}\right)$. Also let $G_{r}$ be the product of Grassmannians $\prod_{j=1}^{r} \operatorname{Gr}(\chi-j, \chi)$. Then $G$ acts on $\tilde{R}, Z$, and $G_{r}$, and there is a $G$-morphism $\tilde{T}: \tilde{R} \rightarrow Z \times G_{r}$, and a family $L\left(\ell_{j}\right)$ of fractional linearizations on $Z \times G_{r}$ depending affinely on $\left(\ell_{j}\right)$, such that $\tilde{T}^{-1}\left(Z \times G_{r}\right)^{s s}\left(\ell_{j}\right)=\tilde{R}^{s s}\left(\ell_{j}\right)$. Moreover, the restriction $\tilde{T}: \tilde{R}^{s s}\left(\ell_{j}\right) \rightarrow\left(Z \times G_{r}\right)^{s s}\left(\ell_{j}\right)$ is finite. The existence of a good quotient $M\left(\ell_{j}\right)$ again follows from the lemma.

In fact, we can say more.

(7.1) Proposition. $T$ is an embedding.

Proof. Since $T$ is injective $[12,4.3]$, it suffices to show its derivative is everywhere injective. At a quotient $\phi: \mathcal{O}^{\chi} \rightarrow E$, the tangent space to Quot is given by the hypercohomology group $\mathbb{H}^{1}$ (End $E \stackrel{\phi}{\rightarrow} E \otimes \mathcal{O}^{\chi}$ ) (cf. [2, 27]). Since $H^{1}\left(E \otimes \mathcal{O}^{\chi}\right)=k^{\chi} \otimes H^{1}(E)=0$, this surjects onto $H^{1}$ (End $\left.E\right)$, and hence onto $H^{1}(\mathcal{O})$, which is the tangent space to $\operatorname{Pic}^{d} C$. So it suffices to show the kernel of this surjection injects into the tangent space to $\mathbb{P} \operatorname{Hom}\left(H^{0}\left(\Lambda^{r} \mathcal{O}^{\chi}\right), H^{0}\left(\Lambda^{r} E\right)\right)$. But the kernel is isomorphic to $\mathbb{H}^{1}\left(\operatorname{End}_{0} E \stackrel{\phi}{\rightarrow} E \otimes \mathcal{O}^{\chi}\right)$, where $\operatorname{End}_{0}$ denotes trace-free endomorphisms, modulo the 1-dimensional subspace generated by $\phi$; this follows from the hypercohomology long exact sequence of the complex

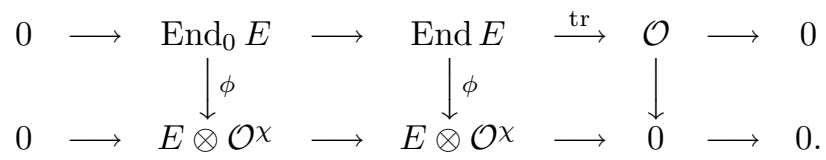

So the kernel injects as desired if and only if the natural map

$$
\mathbb{H}^{1}\left(\text { End } E \stackrel{\phi}{\rightarrow} E \otimes \mathcal{O}^{\chi}\right) \rightarrow \operatorname{Hom}\left(H^{0}\left(\Lambda^{r} \mathcal{O}^{\chi}\right), H^{0}\left(\Lambda^{r} E\right)\right)
$$

is injective as well.

What is this natural map? It is obtained from the derivative of $T$; since $T$ is essentially $\phi \mapsto \Lambda^{r} \phi$, the derivative of $T$ at $\phi$ is essentially $\psi \mapsto\left(\Lambda^{r-1} \phi\right) \wedge \psi$. More precisely, an element of the hypercohomology group above is determined by Čech cochains $g \in C^{1}\left(\operatorname{End}_{0} E\right)$ and $\psi \in C^{0}\left(E \otimes \mathcal{O}^{\chi}\right)$ such that $g \phi=d \psi$. Since $\phi$ is surjective, the hypercohomology class of the pair is uniquely determined by $\psi$; on the other hand, a cochain $\psi$ determines the trivial hypercohomology class if and only if $\psi=f \phi$ for some $f \in C^{0}\left(\operatorname{End}_{0} E\right)$. The natural map to $\operatorname{Hom}\left(H^{0}\left(\Lambda^{r} \mathcal{O}^{\chi}\right), H^{0}\left(\Lambda^{r} E\right)\right)$ is then indeed given by $\psi \mapsto\left(\Lambda^{r-1} \phi\right) \wedge \psi$; its injectivity follows from the lemma below.

(7.2) Lemma. If $\phi: k^{\chi} \rightarrow k^{r}$ is a linear surjection of vector spaces, and $\psi: k^{\chi} \rightarrow$ $k^{r}$ is a linear map, then $\left(\Lambda^{r-1} \phi\right) \wedge \psi=0$ if and only if $\psi=f \phi$ for some $f \in$ End $_{0} k^{r}$. 
Proof. Suppose first that $\chi=r$. Then $\phi$ is invertible, and $\left(\Lambda^{r-1} \phi\right) \wedge \psi$ is a homomorphism of 1-dimensional vector spaces. Indeed, if $e_{1}, \ldots, e_{r}$ is the standard basis for $k^{r}$, then $\Lambda^{r} k^{\chi}$ is spanned by $\phi^{-1} e_{1} \wedge \cdots \wedge \phi^{-1} e_{r}$. But

$$
\begin{aligned}
& \left(\left(\Lambda^{r-1} \phi\right) \wedge \psi\right)\left(\phi^{-1} e_{1} \wedge \cdots \wedge \phi^{-1} e_{r}\right) \\
& \quad=\sum_{i} e_{1} \wedge \cdots \wedge e_{i-1} \wedge \psi \phi^{-1}\left(e_{i}\right) \wedge e_{i+1} \wedge \cdots \wedge e_{r} \\
& \quad=\left(\operatorname{tr} \psi \phi^{-1}\right)\left(e_{1} \wedge \cdots \wedge e_{r}\right),
\end{aligned}
$$

so the lemma is true when $\chi=r$. In the general case, $\left(\Lambda^{r-1} \phi\right) \wedge \psi=0$ implies $\operatorname{ker} \psi \supset \operatorname{ker} \phi$, for if not, let $\phi^{-1}$ be a right inverse for $\phi$, and let $u \in \operatorname{ker} \phi \backslash \operatorname{ker} \psi$. For some $i$, the coefficient of $e_{i}$ in $\psi(u)$ is nonzero. Then

$$
\begin{aligned}
\left(\left(\Lambda^{r-1} \phi\right) \wedge \psi\right)\left(\phi^{-1} e_{1} \wedge \cdots \wedge \phi^{-1} e_{i-1}\right. & \left.\wedge u \wedge \phi^{-1} e_{i+1} \wedge \cdots \wedge \phi^{-1} e_{r}\right) \\
& =e_{i} \wedge \cdots \wedge e_{i-1} \wedge \psi(u) \wedge e_{i+1} \wedge \cdots \wedge e_{r} \neq 0 .
\end{aligned}
$$

So $\psi$ descends to $k^{\chi} / \operatorname{ker} \phi$; this has dimension $r$, so the case above applies.

(7.3) Corollary. $\tilde{T}$ is an embedding.

Proof. $\tilde{R}$ is a bundle of flag varieties over $R$, and each fiber clearly embeds in $G_{r}$.

Let $X$ be the Zariski closure in $Z \times G_{r}$ of $\tilde{T}(\tilde{R})$.

(7.4) Corollary. The moduli space $M\left(\ell_{j}\right)$ of semistable parabolic bundles is $X / / G\left(\ell_{j}\right)$.

Proof. Since $\tilde{T}^{-1}\left(Z \times G_{r}\right)^{s s}\left(\ell_{j}\right)=\tilde{R}^{s s}\left(\ell_{j}\right)$, this is automatic provided there are no semistable points in $X \backslash \tilde{T}(\tilde{R})$. Since $M\left(\ell_{j}\right)$ is already projective, any such points would be in the orbit closures of semistable points in $\tilde{T}(\tilde{R})$. Hence there would be $x \in \tilde{R}^{s s}\left(\ell_{j}\right)$, and a 1-parameter subgroup $\lambda(t) \subset G$, such that $\lim \lambda(t) \cdot x \notin \tilde{T}(\tilde{R})$, but $\mu^{\ell_{j}}(\tilde{T}(x), \lambda)=0$, where $\mu^{\ell_{j}}$ is the valuation used in the numerical criterion [24, 4.8; GIT, Defn. 2.2]. But all the destabilizing subgroups of points in $\tilde{T}(\tilde{R})$ correspond to destabilizing subbundles, and their limits are points corresponding to the associated graded subbundles; in particular, they are in $\tilde{T}(\tilde{R})$.

We are therefore in a position to apply our main results. Let us first look for walls and chambers. Notice that the stability condition only changes at values where there can exist subbundles whose parabolic slope equals that of $E$. If such a subbundle has rank $r^{+}$, degree $d^{+}$, and weights $\ell_{j_{i}^{+}}$for some $\left\{j_{i}^{+}\right\} \subset\{1, \ldots, r\}$, then the slope condition is

$$
\frac{d^{+}+\sum_{i=1}^{r^{+}} \ell_{j_{i}^{+}}}{r^{+}}=\frac{d+\sum_{j=1}^{r} \ell_{j}}{r} .
$$

This determines a codimension 1 affine subset of $W$, which is one of our walls. The complementary numbers $r^{-}=r-r^{+}, d^{-}=d-d^{+}$, and $\left\{\ell_{j_{i}^{-}}\right\}=\left\{\ell_{j}\right\} \backslash\left\{\ell_{j_{i}^{+}}\right\}$of course determine the same wall, but no other numbers do. Also, there are only finitely many walls, since for a given $r^{+}$and $\left\{\ell_{j_{i}^{+}}\right\}$, the affine hyperplane defined by the above equation only intersects $W$ for finitely many $d^{+}$. The connected components of the complement of the walls are the chambers; for purely numerical reasons, the semistability condition is constant in the interior of a chamber. 
Now, as in the set-up of $\S 3$, suppose $\left(\ell_{j}\right)$ lies on a single wall in $W$, and choose $\left(\ell_{j}^{+}\right)$and $\left(\ell_{j}^{-}\right)$in the adjacent chambers such that the line segment connecting them crosses a wall only at $\left(\ell_{j}\right)$. Then $x \in X$ belongs to $X^{0}$ if and only if it represents a parabolic bundle which splits as $E_{+} \oplus E_{-}$, where $E_{ \pm}$are $\left(\ell_{j_{i}^{ \pm}}\right)$-stable parabolic bundles. This is because, to be in $X^{s s}(0) \backslash X^{s s}(+)$, a parabolic bundle must have a semistable parabolic subbundle $E_{+}$of rank $r^{+}$, degree $d^{+}$, and weights $\ell_{j_{i}^{+}}$. Indeed, $E_{+}$must be stable, for since $\left(\ell_{j}\right)$ lies on only one wall, $E_{+}$can have no parabolic subbundle of the same slope. For the same reason $E / E_{+}$must be stable. On the other hand, to be in $X^{s s}(0) \backslash X^{s s}(-), E$ must have a stable parabolic subbundle $E_{-}$of rank $r^{-}$, degree $d^{-}$, and weights $\ell_{j_{i}^{-}}$. Since all the weights are distinct, $E_{-}$ cannot be isomorphic to $E_{+}$; so there is a nonzero map $E_{-} \rightarrow E / E_{+}$. By [26, III Prop. 9(c)], this map must be an isomorphism, so $E$ splits as $E_{+} \oplus E_{-}$.

On the other hand, if $E_{+}$and $E_{-}$are any stable parabolic bundles with rank, degree, and weights as above, then $E_{+} \oplus E_{-}$is certainly represented in $X^{0}$. Hence $X^{0} / / G(0)=M\left(\ell_{j_{i}^{+}}\right) \times M\left(\ell_{j_{i}^{-}}\right)$, the product of two smaller moduli spaces.

It is now easy to verify the hypotheses of our strongest result (4.8). First, $X$ is smooth on $X^{s s}\left(\ell_{j}\right)$, hence at $X^{0}$. Second, for any $x \in X^{0}$, the stabilizer $G_{x}$ is the subgroup isomorphic to $k^{\times}$acting on $H^{0}\left(E_{+}\right)$with weight $\chi^{-} / c$ and on $H^{0}\left(E_{-}\right)$ with weight $-\chi^{+} / c$, where $c$ is the greatest common divisor $\left(\chi^{+}, \chi^{-}\right)$. This is because any $g \in \mathrm{GL}(\chi)$ stabilizing a point in $X^{0}$ induces an automorphism of $\mathbf{E}_{x}$, and vice versa, so for $x \in X^{0}$ there is an isomorphism $\mathrm{GL}(\chi)_{x} \cong \operatorname{Par} \operatorname{Aut}\left(E_{+} \oplus E_{-}\right)=$ $k^{\times} \times k^{\times}$; but only the automorphisms acting with the weights above correspond to special linear $g$. Third, if $L_{j}$ is the ample generator of $\operatorname{Pic} \operatorname{Gr}(\chi-j, \chi)$, then for $x \in X^{0}, G_{x} \cong k^{\times}$acts on $\left(L_{j}\right)_{x}$ with weight $\left(n_{j}^{+} \chi^{-}-n_{j}^{-} \chi^{+}\right) / c$, where $n_{j}^{ \pm}$are the number of $j_{i}^{ \pm}$less than or equal to $j$. But since $\chi^{+} / c$ and $\chi^{-} / c$ are coprime, so are these weights for some two values of $j$. Bhosle gives a formula for the linearization on $Z \times$ Gr determined by $\left(\ell_{j}\right)$ in terms of the $L_{i}$; an easy argument using this formula shows that $\left(\ell_{j}^{ \pm}\right)$can be chosen within their chambers so that $G_{x}$ acts with coprime weights on the corresponding linearizations.

Because all semistable parabolic bundles are represented by points in $X$, and because semistability is an open condition, the universal family of parabolic bundles is a versal family near any point $x \in X^{0}$. Moreover, two points in $X$ represent the same parabolic bundle if and only if they are in the same orbit. The normal bundle $N_{G \cdot x / X}$ to an orbit is therefore exactly the deformation space of the parabolic bundle. For $\mathbf{E}_{x}=E_{+} \oplus E_{-}$as above this is $H^{1}\left(X, \operatorname{Par} \operatorname{End}\left(E_{+} \oplus E_{-}\right)\right)$. The stabilizer $G_{x}$ acts with weight $r^{ \pm} /\left(r^{+}, r^{-}\right)$on $E_{ \pm}$, so $N^{0}=H^{1}\left(X, \operatorname{Par}\right.$ End $\left.E_{+}\right) \oplus$ $H^{1}\left(X, \operatorname{Par} E n d E_{-}\right)$, and $N^{ \pm}=H^{1}\left(X, \operatorname{Par} \operatorname{Hom}\left(E_{\mp}, E_{ \pm}\right)\right)$. Moreover, every element in $N^{ \pm}$is acted on with weight exactly $\pm\left(\chi^{+}+\chi^{-}\right) / c$, so $N^{ \pm}$descend to vector bundles $W^{ \pm}$over $M\left(\ell_{j_{i}^{+}}\right) \times M\left(\ell_{j_{i}^{-}}\right)$. Indeed, if $\mathbf{E}_{ \pm} \rightarrow M\left(\ell_{j_{i}^{ \pm}}\right) \times C$ are universal bundles, then $W^{ \pm}=\left(R^{1} \pi\right) \operatorname{Par} \operatorname{Hom}\left(\mathbf{E}_{\mp}, \mathbf{E}_{ \pm}\right)$. Theorem (4.8) then states that $\mathbb{P} W^{ \pm}$are the exceptional loci of the morphisms $M\left(\ell_{j}^{ \pm}\right) \rightarrow M\left(\ell_{j}\right)$. This is the result of Boden and $\mathrm{Hu}$ [8]. Moreover, (4.8) asserts that the blow-ups of $M\left(\ell_{j}^{ \pm}\right)$at $\mathbb{P} W^{ \pm}$, and of $M\left(\ell_{j}\right)$ at $M\left(\ell_{j_{i}^{+}}\right) \times M\left(\ell_{j_{i}^{-}}\right)$, are all naturally isomorphic to the fibred product $M\left(\ell_{j}^{-}\right) \times_{M\left(\ell_{j}\right)} M\left(\ell_{j}^{+}\right)$.

With the obvious modifications, the same techniques and results go through for bundles with parabolic structure at several marked points, or with degenerate flags. 


\section{BRADLOW PAIRS}

The moduli spaces of Bradlow pairs on our curve $C$ can be studied in the same way. The role of the weights will be played by a positive parameter $\sigma \in \mathbb{Q}$. Our goal will be to reprove the main result of $\S 3$ in [27], which constructed an explicit sequence of blow-ups and blow-downs between projective space and a variety surjecting to the moduli space of stable rank 2 bundles on $C$. We will also reprove the analogous result of Bertram, Daskalopoulos, and Wentworth [2] on the Quot scheme.

A Bradlow pair is a pair $(E, \phi)$ consisting of a vector bundle $E$ over $C$ and a nonzero section $\phi \in H^{0}(X, E)$. We refer to $[5,6,27]$ for basic definitions and results on Bradlow pairs. As in [27], we confine ourselves to the study of rank 2 pairs. In this case a Bradlow pair of degree $d$ is $\sigma$-semistable if for all line bundles $L \subset E$,

$$
\begin{array}{ll}
\operatorname{deg} L \leq d / 2-\sigma & \text { if } \phi \in H^{0}(L), \text { and } \\
\operatorname{deg} L \leq d / 2+\sigma & \text { if } \phi \notin H^{0}(L) .
\end{array}
$$

It is $\sigma$-stable if both inequalities are strict.

The moduli spaces $B_{d}(\sigma)$ of $\sigma$-semistable rank 2 pairs were constructed in [27]. In that paper, the determinant was fixed, but to parallel the discussion of parabolic bundles above we shall now allow arbitrary determinant. With that modification, the construction goes as follows.

It suffices to construct $B_{d}(\sigma)$ for $d$ sufficiently large. This is because, for any effective divisor $D, B_{d}(\sigma)$ will be embedded in $B_{d+2|D|}(\sigma)$ as the locus where $\phi$ vanishes on $D$. So assume that $d / 2-\sigma>2 g-2$, and let $\chi=d+2(1-g)$. Let Quot, $R$ and $Z$ be as in $\S 7$ above, and let $G=\operatorname{SL}(\chi)$ act diagonally on $R \times \mathbb{P}^{\chi-1}$. The hypothesis $d / 2-\sigma>2 g-2$ implies that every $\sigma$-semistable pair is represented by a point in $R \times \mathbb{P}^{\chi-1}$. Let $\left(Z \times \mathbb{P}^{\chi-1}\right)^{s s}(\sigma)$ denote the semistable set with respect to the fractional linearization $\mathcal{O}(\chi+2 \sigma, 4 \sigma)$, and let $\left(R \times \mathbb{P}^{\chi-1}\right)^{s s}(\sigma)$ denote the $\sigma$-semistable set in the sense of the definition above. Then the natural $G$-morphism $T \times 1: R \times \mathbb{P}^{\chi-1} \rightarrow Z \times \mathbb{P}^{\chi-1}$ satisfies $T^{-1}\left(Z \times \mathbb{P}^{\chi-1}\right)^{s s}(\sigma)=$ $\left(R \times \mathbb{P}^{\chi-1}\right)^{s s}(\sigma)$. Moreover, by (7.1), it is an embedding. So if $X$ denotes the Zariski closure of its image, then for reasons like those given in $\S 7$, the moduli space $B_{d}(\sigma)$ is the geometric invariant theory quotient $X / / G(\sigma)$, where $\sigma$ denotes the fractional linearization $T^{*} \mathcal{O}(\chi+2 \sigma, 4 \sigma)$.

So again our main results apply. The stability condition only changes for $\sigma \in$ $d / 2+\mathbb{Z}$, so these points are the walls. Fix one such $\sigma$. Then $x \in X$ belongs to $X^{0}$ if and only if it represents a pair which splits as $L \oplus M$, where $\operatorname{deg} L=d / 2-\sigma$ and $\phi \in H^{0}(L)$. Indeed, a subbundle $L$ of degree $d / 2-\sigma$ is needed to violate the first semistability condition to the right of the wall, and a subbundle $M$ of degree $d / 2+\sigma$ is needed to violate the second semistability condition to the left. But since $\operatorname{deg} M=\operatorname{deg} E / L$, the map $M \rightarrow E / L$ is an isomorphism, so $E$ is split. On the other hand, for any pair $(L, \phi)$ with $\operatorname{deg} L=d / 2-\sigma$ and $\phi \in H^{0}(L) \backslash 0$, and for any line bundle $M$ with $\operatorname{deg} M=d / 2+\sigma$, certainly $(L \oplus M, \phi \oplus 0)$ is represented in $X^{0}$. Hence $X^{0} / / G(0)=S^{i} C \times \operatorname{Pic}^{d-i} C$, where $i=d / 2-\sigma$ and $S^{i} C$ is the $i$ th symmetric product.

It is now easy to verify the hypotheses of our strongest result (4.8). First, $X$ is smooth at $X^{s s}(0)$, hence at $X^{0}$. Second, for any $x \in X^{0}$, the stabilizer $G_{x}$ is the subgroup isomorphic to $k^{\times}$acting on $H^{0}(L)$ with weight $\chi(M) / c$ and on $H^{0}(M)$ with weight $-\chi(L) / c$, where $c$ is the greatest common divisor $(\chi(L), \chi(M))$. This 
is because any $g \in \mathrm{GL}(\chi)$ stabilizing a point in $X^{0}$ induces an automorphism of the corresponding pair, and vice versa, but only the automorphisms acting with the weights above correspond to special linear $g$. Third, this stabilizer $k^{\times}$acts on $\mathcal{O}(1,0)_{x}$ with weight $(\chi(M)-\chi(L)) / c$, and on $\mathcal{O}(0,1)_{x}$ with weight $\chi(M) / c$. These are coprime, so linearizations with coprime weights can be chosen within the chambers adjacent to $\sigma$.

As in $\S 7$, the normal bundle to an orbit is exactly the deformation space of the Bradlow pair. For any pair $(E, \phi)$, this is the hypercohomology group $\mathbb{H}^{1}($ End $E \stackrel{\phi}{\rightarrow} E)$. (See [6] or [27, (2.1)]; the slightly different formula in [27] arises because the determinant is fixed.) More naturally, the term $E$ in the complex is actually $E \otimes \mathcal{O}$, where $\mathcal{O}$ is the dual of the subsheaf of $E$ generated by $\phi$; it is acted on accordingly by $G$. For a pair $(L \oplus M, \phi \oplus 0)$ represented in $X^{0}$, this splits as

$$
\mathbb{H}^{1}(\mathcal{O} \oplus \mathcal{O} \stackrel{\phi \oplus 0}{\longrightarrow} L) \oplus \mathbb{H}^{1}\left(L M^{-1} \rightarrow 0\right) \oplus \mathbb{H}^{1}\left(M L^{-1} \stackrel{\phi}{\rightarrow} M\right) .
$$

These are acted on by the stabilizer $k^{\times}$with weights 0 and $\pm(\chi(M)+\chi(L)) / c$ respectively, so they are exactly $N^{0}, N^{+}$, and $N^{-}$. The expressions for $N^{ \pm}$can be simplified: $\mathbb{H}^{1}\left(L M^{-1} \rightarrow 0\right)$ is just $H^{1}\left(L M^{-1}\right)$, and if $D$ is the divisor of zeroes of $\phi$, the long exact sequence of

$$
0 \longrightarrow M L^{-1} \longrightarrow M \longrightarrow \mathcal{O}_{D} \otimes M \longrightarrow 0
$$

implies that $\mathbb{H}^{1}\left(M L^{-1} \stackrel{\phi}{\rightarrow} M\right)$ is just $H^{0}\left(\mathcal{O}_{D} \otimes M\right)$. Since every element in $N^{ \pm}$ is acted on with weight exactly $\pm(\chi(M)+\chi(L)) / c, N^{ \pm}$descend to vector bundles $W^{ \pm}$over $S^{i} C \times \mathrm{Pic}^{d-i} C$. Indeed, if $\mathbf{M} \rightarrow \mathrm{Pic}^{d-i} C \times C$ is a Poincaré bundle and $\Delta \subset S^{i} C \times C$ is the universal divisor, then $W^{+}=\left(R^{1} \pi\right) \mathbf{M}^{-1}(\Delta)$ and $W^{-}=$ $\left(R^{0} \pi\right) \mathcal{O}_{\Delta} \otimes \mathbf{M}$.

Theorem (4.8) then states that $\mathbb{P} W^{ \pm}$are the exceptional loci of the morphisms $B_{d}\left(\sigma \pm \frac{1}{2}\right) \rightarrow B_{d}(\sigma)$, and that the blow-ups of $B_{d}\left(\sigma \pm \frac{1}{2}\right)$ at $\mathbb{P} W^{ \pm}$, and of $B_{d}(\sigma)$ at $S^{i} C \times \operatorname{Pic}^{d-i} C$, are all isomorphic to the fibred product $B_{d}\left(\sigma-\frac{1}{2}\right) \times_{B_{d}(\sigma)} B_{d}\left(\sigma+\frac{1}{2}\right)$. This includes the main result (3.18) of [27]; to recover the $W^{ \pm}$obtained there for a fixed determinant line bundle $\Lambda$, just substitute $\mathbf{M}=\Lambda(-\Delta)$.

Notice that since the construction only works for $d$ large, the result has so far only been proved in that case. For general $d$, choose disjoint divisors $D, D^{\prime}$ of degree $|D|=\left|D^{\prime}\right|$ such that $d+2|D|$ is large enough. Then $D$ and $D^{\prime}$ determine two different embeddings $B_{d+2|D|}(\sigma) \rightarrow B_{d+4|D|}(\sigma)$ whose images intersect in $B_{d}(\sigma)$. The result for $B_{d}(\sigma)$ then follows readily from the result for $B_{d+2|D|}(\sigma)$ and $B_{d+4|D|}(\sigma)$.

A similar argument proves the analogous result of Bertram, Daskalopoulos and Wentworth [2] on Bradlow $n$-pairs. These are pairs $(E, \phi)$, where $E$ is as before, but $\phi$ is now a nonzero element of $H^{0}\left(E \otimes \mathcal{O}^{n}\right)$. The stability condition is just like that for ordinary Bradlow pairs, except that the two cases are $\phi \in H^{0}\left(L \otimes \mathcal{O}^{n}\right)$ and $\phi \notin H^{0}\left(L \otimes \mathcal{O}^{n}\right)$. There is no geometric invariant theory construction in the literature of moduli spaces of $n$-pairs, but the construction of [27] for 1-pairs generalizes in the obvious way; for example, $\mathbb{P}^{\chi^{-1}}$ gets replaced by $\mathbb{P}^{n \chi-1}$.

Again the stability condition only changes for $\sigma \in d / 2+\mathbb{Z}$, so fix one such $\sigma$. Assume that $d / 2-\sigma>2 g-2$, so that the moduli space can be constructed directly as a geometric invariant theory quotient. Then $x \in X^{0}$ if and only if it splits as $L \oplus M$, where $\operatorname{deg} L=i=d / 2-\sigma$ and $\phi \in H^{0}\left(L \otimes \mathcal{O}^{n}\right)$. Hence $X^{0} / / G(0)=\mathbb{P} U \times \operatorname{Pic}^{d-i} C$, where $U=\left(R^{0} \pi\right)\left(\mathbf{L} \otimes \mathcal{O}^{n}\right)$ for some Poincaré bundle $\mathbf{L}$ over $\operatorname{Pic}^{i} C \times C$. The hypotheses of (4.8) are verified exactly as before. The normal bundle to an orbit 
is again the deformation space, but this is now $\mathbb{H}^{1}$ (End $\left.E \stackrel{\phi}{\rightarrow} E \otimes \mathcal{O}^{n}\right)$; for a pair $(L \oplus M, \phi \oplus 0)$ represented in $X^{0}$, this splits as

$$
\mathbb{H}^{1}\left(\mathcal{O} \oplus \mathcal{O} \stackrel{\phi \oplus 0}{\longrightarrow} L \otimes \mathcal{O}^{n}\right) \oplus \mathbb{H}^{1}\left(L M^{-1} \rightarrow 0\right) \oplus \mathbb{H}^{1}\left(M L^{-1} \stackrel{\phi}{\rightarrow} M \otimes \mathcal{O}^{n}\right) .
$$

Again these are exactly $N^{0}, N^{+}$, and $N^{-}$. The expression for $N^{+}$is simply $H^{1}\left(L M^{-1}\right)$, but the expression for $N^{-}$cannot be simplified very much. If $F$ is defined to be the cokernel of the sheaf injection $M L^{-1} \rightarrow M \otimes \mathcal{O}^{n}$ induced by $\phi$, then $N^{-}=H^{0}(F)$, but this is not very helpful as $F$ may not be locally free. In any case, $N^{ \pm}$descend as before, to vector bundles $W^{ \pm}$constructed as follows. There is a tautological element of $H^{0}\left(\operatorname{Pic}^{i} C\right.$; End $\left.U\right)=H^{0}\left(\operatorname{Pic}^{i} C \times C ; U^{*} \otimes \mathbf{L} \otimes \mathcal{O}^{n}\right)=$ $H^{0}\left(\mathbb{P} U \times C ; \mathcal{O}(1) \otimes \mathbf{L} \otimes \mathcal{O}^{n}\right)$, so the vector bundle $\mathcal{O}(1) \otimes \mathbf{L} \otimes \mathcal{O}^{n}$ has a tautological section on $\mathbb{P} U \times C$. There is therefore a tautological sheaf injection $\mathbf{M L}^{-1} \rightarrow \mathcal{O}(1) \otimes \mathbf{M} \otimes \mathcal{O}^{n}$ on $\mathbb{P} U \times \mathrm{Pic}^{d-i} C \times C$, where $\mathbf{M}$ is a Poincaré bundle on $\mathrm{Pic}^{d-i} C \times C$. Let $\mathbf{F}$ be the cokernel of this injection; then $W^{-}=\left(R^{0} \pi\right) \mathbf{F}$. As before, $W^{+}=\left(R^{1} \pi\right) \mathbf{L M}^{-1}$.

Theorem (4.8) then gives a result precisely analogous to the one stated above for 1-pairs. However, the argument passing from large $d$ to general $d$ no longer works, so this result is only valid for $i=d / 2-\sigma>2 g-2$. Indeed, for $i$ smaller than this, $U$ is not locally free, and $X^{0} / / G(0)$ has bad singularities. Nevertheless, Bertram et al. succeed in using this result to compute certain Gromov invariants of Grassmannians.

\section{ACKNOWLEDGEMENTS}

I am grateful to Aaron Bertram, Igor Dolgachev, David Eisenbud, Jack Evans, Antonella Grassi, Stuart Jarvis, Yi Hu, János Kollár, Kenji Matsuki, Rahul Pandharipande, David Reed, Eve Simms, and especially Frances Kirwan, Peter Kronheimer, and Miles Reid for very helpful conversations and advice.

\section{REFERENCES}

1. M.F. Atiyah, On analytic surfaces with double points, Proc. R. Soc. Lond. A 247 (1958) 237-44. MR 20:2472

2. A. Bertram, G. Daskalopoulos, and R. Wentworth, Gromov invariants for holomorphic maps from Riemann surfaces to Grassmannians, J. Amer. Math. Soc., to appear.

3. U.N. Bhosle, Parabolic vector bundles on curves, Arkiv för Mat. 27 (1989) 15-22. MR 90f: 14007

4. A. Bialynicki-Birula, Some theorems on actions of algebraic groups, Ann. Math. 98 (1973) 480-497. MR 51:3186

5. S. Bradlow, Special metrics and stability for holomorphic bundles with global sections, J. Diff. Geom. 33 (1991) 169-213. MR 91m:32031

6. S. Bradlow and G. Daskalopoulos, Moduli of stable pairs for holomorphic bundles over Riemann surfaces, Int. J. Math. 2 (1991) 477-513. MR 93b:58026

7. M. Brion and C. Procesi, Action d'un tore dans une variété projective, Operator algebras, unitary representations, enveloping algebras, and invariant theory, (A. Connes, M. Duflo, A. Joseph, and R. Rentschler, eds.), Birkhauser, 1990, pp. 509-539. MR 92m:14061

8. H. Boden and Y. Hu, Variation of moduli of parabolic bundles, Math. Ann., to appear.

9. I. Dolgachev and Y. Hu, Variation of geometric invariant theory quotients, preprint.

10. J.-M. Drezet and M.S. Narasimhan, Groupe de Picard des variétés de modules de fibrés semistables sur les courbes algébriques, Inv. Math. 97 (1989) 53-94. MR 90d:14008

11. J.J. Duistermaat and G.J. Heckman, On the variation in the cohomology of the symplectic form of the reduced phase space, Inv. Math. 69 (1982) 259-268. Addendum: 72 (1983) 153158. MR 84h:58051a,b 
12. D. Gieseker, On the moduli of vector bundles on an algebraic surface, Ann. of Math. 106 (1977) 45-60. MR 81h:14014

13. A. Grassi and J. Kollár, Log canonical models, Astérisque 211 (1992) 29-45. MR 94f:14013

14. A. Grothendieck, Sur quelques points d'algèbre homologique, Tôhoku Math. J. 9 (1957) 119221. MR 21:1328

15. A. Grothendieck, Technique de descente et théorèmes d'existence en géométrie algébrique, IV: Les schémas de Hilbert, Sém. Bourbaki 1960-61, Exp. 221; reprinted in Fondements de la géométrie algébrique, Secrétariat Math., Paris, 1962. MR 26:3566

16. R. Hartshorne, Algebraic geometry, Springer-Verlag, 1977. MR 57:3116

17. Y. Hu, The geometry and topology of quotient varieties of torus actions, Duke Math. J. 68 (1992) 151-184. Erratum: 68 (1992) 609. MR 93k:14019a,b

18. F.C. Kirwan, Cohomology of quotients in algebraic and symplectic geometry, Princeton, 1984. MR 86i:58050

19. D. Luna, Slices étales, Mém. Soc. Math. France 33 (1973) 81-105. MR 49:7269

20. I.G. Macdonald, Symmetric products of an algebraic curve, Topology 1 (1962) 319-343. MR 27:1445

21. V.B. Mehta and C.S. Seshadri, Moduli of vector bundles on curves with parabolic structures, Math. Ann. 248 (1980) 205-239. MR 81i:14010

22. S. Mori, Flip theorem and the existence of minimal models for 3-folds, J. Amer. Math. Soc. 1 (1988) 117-253. MR 89a:14048

23. D. Mumford and J. Fogarty, Geometric invariant theory, second enlarged edition, SpringerVerlag, 1982. MR 86a:14006

24. P.E. Newstead, Introduction to moduli problems and orbit spaces, Tata Inst., Bombay, 1978. MR 81k:14002

25. C.S. Seshadri, Quotient spaces modulo reductive algebraic groups, Ann. of Math. 95 (1972) 511-556. MR 46:9044

26. C.S. Seshadri, Fibrés vectoriels sur les courbes algébriques, Astérisque 96 (1982). MR 85b: 14023

27. M. Thaddeus, Stable pairs, linear systems and the Verlinde formula, Inv. Math. 117 (1994) 317-353. MR 95e:14006

28. M. Thaddeus, Toric quotients and flips, Proceedings of the 1993 Taniguchi symposium, (K. Fukaya, M. Furuta, and T. Kohno, eds.), World Scientific, 1994, pp. 193-213. MR 96b:14067

ABstract. We study the dependence of geometric invariant theory quotients on the choice of a linearization. We show that, in good cases, two such quotients are related by a flip in the sense of Mori, and explain the relationship with the minimal model program. Moreover, we express the flip as the blowup and blow-down of specific ideal sheaves, leading, under certain hypotheses, to a quite explicit description of the flip. We apply these ideas to various familiar moduli problems, recovering results of Kirwan, Boden-Hu, BertramDaskalopoulos-Wentworth, and the author. Along the way we display a chamber structure, following Duistermaat-Heckman, on the space of all linearizations. We also give a new, easy proof of the Bialynicki-Birula decomposition theorem.

St. John's College, Oxford, England

Current address: Department of Mathematics, Harvard University, 1 Oxford St., Cambridge, Massachusetts 02138

E-mail address: thaddeus@math.harvard.edu 